\title{
Localising Cetacean Sounds for the Real-Time Mitigation and Long-Term Acoustic Monitoring of Noise
}

\author{
Michel André, Ludwig Houégnigan, Mike van der Schaar, \\ Eric Delory, Serge Zaugg, Antonio M. Sánchez and Alex Mas \\ Laboratory of Applied Bioacoustics, Technical University of Catalonia, \\ 08800 Vilanova i la Geltrú, Barcelona \\ Spain
}

\section{Introduction}

Noise can have a detrimental effect on cetaceans, as well as on other marine animal species. It can cause stress and increase risk of mortality by interfering with their use of sounds in communication (social behaviour and reproduction) and in navigation (echolocation or biosonar to orientate and look for food). Acoustic overexposure, e.g. in areas of heavy shipping, seismic surveys, military exercises, offshore windmills or gas/oil exploration, can lead to hearing loss. While temporary threshold shift (TTS) represents a reversible hearing loss over time, a permanent threshold shift (PTS) results in non-reversible lesions in mammal ears, i.e. a permanent hearing loss caused by long term and/or intense exposure. Although the impact of low to mid frequency $(<5 \mathrm{kHz})$ acoustic pollution from the above mentioned human marine activities with regard to cetacean disorientation and death remains poorly understood, available evidence is strongly suggestive of some negative direct or indirect effects: There is an increasing mortality rate from shipping collisions, and cetacean mass strandings after military maneuvers have also been recently related with the use of active sonar, both suggesting that some populations may already be suffering from acoustic impact (i.e. TTS, PTS or blast injuries). The control of noise impact on the marine environment constitutes a scientific challenge and requires a dynamic analysis of the situation based on the parallel development of applied solutions to balance human interests and the conservation of marine species. This objective implies the ambitious synthesis of many advanced acoustic technologies that must be designed to monitor the real-time presence of determined cetacean populations in conflictive areas.

Many cetacean species can be identified by their specific calls. The recording of these signature acoustic signals can reveal their presence in monitored areas. Since sound propagates efficiently in water, the detection range of these signals can be quite large, exceeding $100 \mathrm{~km}$ in favourable conditions for low-frequency calls far above visual detection methods. This acoustic potential to non-intrusively detect and monitor cetacean species in their environment gave rise to Passive Acoustic Monitoring (PAM) techniques, for which research is very active. The localisation of whales from their sounds in their habitats was initiated in the 1970s. This was rapidly applied to tracking whales over large distances. 
Advances in electronics, computers and numerical analysis now make this PAM technology more accessible and affordable to small research budgets. Various systems have been used, including radio-linked systems, drifting buoys, and arrays of autonomous recorders for versatile and long-term deployments. The goal of such PAM systems, is the continuous mapping of presence and distribution of whales over ocean basins and assessing their densities, sometimes in quasi real-time. Their performance in effectively accomplishing these tasks depends on the characteristics of the targeted cetacean acoustic signals, the environment, the type of equipment used, its deployment and configuration. This performance may significantly vary from case to case.

However, in any case, PAM's success first depends on the capacity to detect and isolate the target signals from the rest of the sounds in which they are imbedded, especially for distant sources and low signal to noise ratios (SNR). The acoustic signal source level, propagation loss, and local background levels determine detection ranges. Moreover, cetacean sounds vary considerably in time-frequency, from infrasonic calls of baleen whales to ultrasonic clicks of toothed whales, and in amplitudes among species and within a species' vocal repertoire. The ocean noise level also exhibits considerable variability in space and time, in response to fluctuating natural sources, such as wind, ice, rain, sounds produced by various organisms and anthropogenic sources such as shipping. Sound speed structures over the water column can focus sounds from distant sources into sound channels. The 3D spatial arrangements of the sources and the hydrophones are therefore relevant to the PAM configuration.

The Laboratory of Applied Bioacoustics of the Technical University of Catalonia has developed PAM solutions to prevent ship collisions with sperm whales but also to detect, classify and track acoustic sources for the long-term study of noise effects on the marine environment at deep-sea underwater observatories. The first system, called WACS (Whale Anti-Collision System) is a passive system designed to monitor the presence of individual cetaceans or objects and transmit the real-time information of their movements to any ship concerned in preventing possible collisions or harmful operations in areas of interest. The WACS original concept is to instrument a safety corridor for marine mammals, within which cetaceans can be detected, classified, localised and their positions notified to vessels using the corridor to permit timely course alterations. WACS is based on passive acoustics detection and ocean ambient noise to locate and identify the marine mammals present in the survey area. WACS integrates two inter-correlated systems: one 3D listening system called Loc3D which allows the 3D detection of the underwater sound sources (distance, azimuth and elevation), and an azimuthal location system (locAz) of the non vocalizing marine mammals by the spatio-temporal contrast produced by the ambient noise in the survey area. The latter is fundamental, because many of the cetacean species (e.g. sperm and probably beaked whales) are silent while at or near the surface or produce very low frequency sounds difficult to detect above background noise (e.g. most baleen whales species). A simulation tool for 3D acoustic propagation was designed where wideband 3D curved ray solution of the wave equation is implemented. This tool was developed to simulate a bi-static solution formed of an arbitrary number of active acoustic sources, an illuminated object, and a receiver all positioned in 3D space with arbitrary bathymetry. The software recreates the resulting sound mixture of direct, reverberated and echoed signals arriving the array sensors for any array configuration and any number of sources. One object can be placed in the water column and its impact on the acoustic field at the receiver is resolved. The 
software simulations set bounds as for the concept viability. Detection and bearing estimates could be evaluated for vocalising sperm whales.

In addition to the development and use of PAM techniques for mitigation and prevention of ship collisions, the challenge to assess the large-scale influence of artificial noise on marine organisms and ecosystems requires long-term access of this data. Understanding the link between natural and anthropogenic acoustic processes is indeed essential to predict the magnitude and impact of future changes of the natural balance of the oceans. Deep-sea observatories have the potential to play a key role in the assessment and monitoring of these acoustic changes. ESONET is a European Network of Excellence of 12 deep-sea observatories that are deployed from the Arctic to the Gulf of Cadiz (http:/ /www.esonetnoe.org/). ESONET NoE provides data on key parameters from the subsurface down to the seafloor at representative locations and transmits them in real time to shore. The strategies of deployment, data sampling, technological development, standardisation and data management are being integrated with projects dealing with the spatial and near surface time series. LIDO (Listening to the Deep Ocean environment, http://listentothedeep.com) is one of these projects that is allowing the real-time long-term monitoring of marine ambient noise as well as marine mammal sounds in European waters.

In the frame of ESONET and the LIDO project, vocalising sperm whales were detected offshore the port of Catania (Sicily) with a bottom-mounted (around 2080m depth) tetrahedral compact array intended for real-time detection, localisation and classification of cetaceans. Various broadband space-time methods were implemented and permitted to map the sound radiated during the detected clicks and to consequently localise not only sperm whales but also vessels. Hybrid methods were developed as well which permit to make space-time methods more robust to noise and reverberation and moderate computation time. In most cases, the small variance obtained for these estimates reduces the necessity of additional statistical clustering. Consistent tracking of both sperm whales and vessels in the area have validated the performance of the approach.

The development of these techniques that we present here represent a major step forward the mitigation of the effects of invasive sound sources on cetaceans and monitoring the longterm interactions of noise.

\section{The sperm whale sonar}

Sperm whales are known to spend most of their time foraging and feeding on squids at depths of several hundreds of meters where the light is scarce. While foraging, sperm whales produce a series of acoustic signals called 'usual clicks'. The coincidence of the continuous production of usual clicks together with the associated feeding behaviour has led authors to suppose that those specific signals could be involved in the process of detecting prey. Because the usual click has known acoustic signal features differing from most of the described echolocation signals of other species, there has long been speculation about the sperm whale sonar capabilities. While the usual clicks of this species were considered to support mid-range echolocation, no physical characteristics of the signal had, until very recently, clearly confirmed this assumption nor had it been explained how sperm whales forage on low sound reflective bodies like squid. The recent data on sperm whale onaxis recordings have shed some light on those questions and allowed us to perform simulations in controlled environments to verify the possible mid-range sonar function of usual clicks during foraging processes (André et al., 2007, 2009). 
Research on the acoustic features of sperm whale clicks is well documented, but the obtained quantitative results have varied substantially between publications. Only recently have the intricate sound production mechanisms been addressed with reliable quantitative data (Møhl et al., 2003; Zimmer et al., 2005).

\section{Source level and directionality}

In 1980 Watkins reported a source level (SL) of $180 \mathrm{~dB}$ re $1 \mu \mathrm{Pa}-\mathrm{m}$ and suggested that clicks were rather omnidirectional (Watkins, 1980), whereas recent results from Møhl et al. estimate this source level to be as high as $223 \mathrm{dBpeRMS}$ re $1 \mu \mathrm{Pa}-\mathrm{m}$ with high directionality (Møhl et al., 2003). Morphophysiological observations on the unusual shape and weight of the sperm whale nose are in clear agreement with the hypothesis of its highly directional and powerful sonar function, supported by Møhl's results. Goold \& Jones (1995) recorded clicks from both an adult male and female and measured a shift to higher frequencies of the main spectral peaks, from $400 \mathrm{~Hz}$ to $1.2 \mathrm{kHz}$, and $2 \mathrm{kHz}$ to $3 \mathrm{kHz}$, though they noticed that this shift was rather unstable. Spectral contents of clicks as a function of body size and, most importantly, animal orientation information could help to explain this difference in received levels. The almost ubiquitous lack of animal heading information at click recording time in published material makes results hardly usable for a reliable 3D model. To date, Møhl et al. (2003) and Zimmer et al. (2005) are the only studies that provide sufficient calibrated material to produce a correct model. The reported $15 \mathrm{kHz}$ centroïd frequency and apparent source levels higher than $220 \mathrm{dBRMS}$ re $1 \mu \mathrm{Pam}$ corroborate the fact that most previously published click levels and characteristics certainly stemmed from off-axis recordings or unsuitable recording bandwidth. Sperm whale click source level and time-frequency characteristics can be predicted by inferring a threedimensional model, which is based upon well-known physics principles, such as the direct relationship between the size of the sound production apparatus and its directionality (Tucker \& Glazey, 1966).

\section{Click time-frequency characteristics}

Acoustic recordings of distant sperm whales have often revealed the multi-pulsed nature of their clicks, with interpulse intervals that may be related to head size or more specifically the distance between the frontal and distal air sacs situated at both ends of the spermaceti organ (Alder-Frenchel, 1980). While the utility of this multipulsed pattern is unclear, Møhl et al. (2003) have shown that one single main pulse appears for on-axis recordings. They suggest that the radiated secondary pulses are acoustic clutter resulting from the on-axis main pulse generation. This clearly advocates that the animal orientation must be known in order to create a 3D click time-frequency model from recorded sound. These multiple pulses are found in the upper half of the received click spectrum while on-axis recordings reveal a centroïd frequency of $15 \mathrm{kHz}$ and a monopulse pattern (Figure 1). On recordings we performed in the Canary Islands from whales of unknown orientation, more than six secondary pulses could at times be observed. A continuous low frequency part (below 1 $\mathrm{kHz}$ ), which does not seem to follow a repetitive pattern and may last more than $10 \mathrm{~ms}$, has also been documented (Goold \& Jones, 1995; Zimmer et al., 2003). Proper time-frequency modelling from recorded clicks should therefore account for animal instantaneous distance, heading and depth, and environmental conditions with sufficient space-time resolution. To our knowledge, no other report fulfils these requirements. Yet, our aim here will not be to model an even near-perfect click generator, but a system that is in agreement with our current knowledge. 


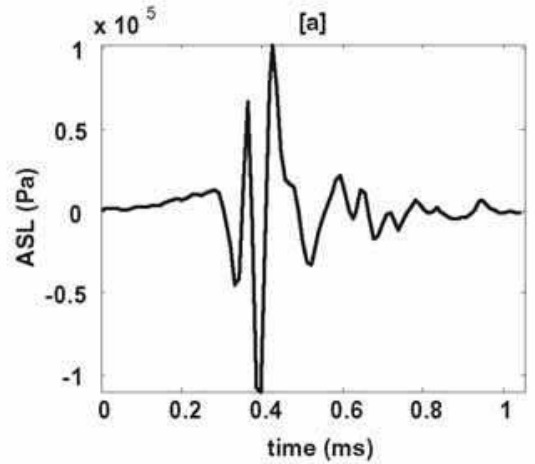

[c]

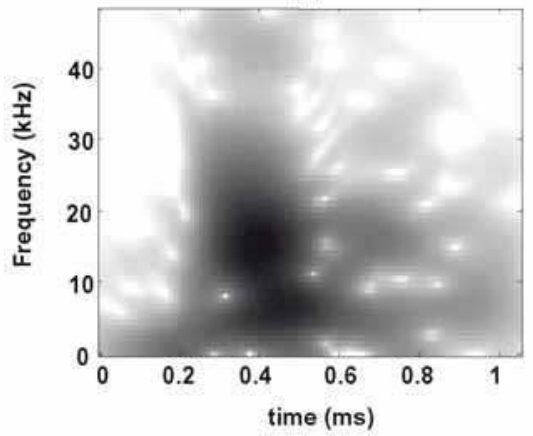

[b]

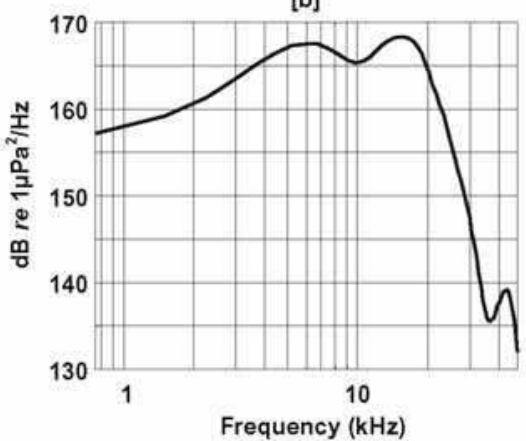

[d]

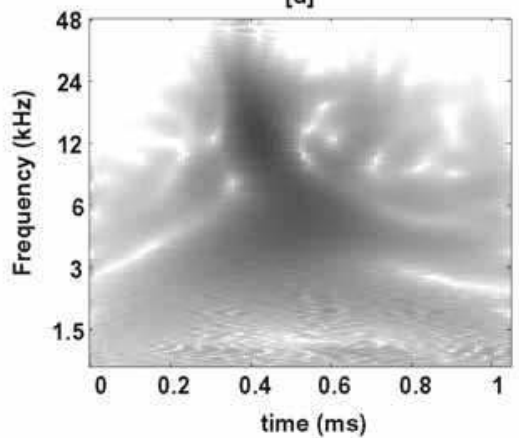

Fig. 1. This monopulse click was recorded near on-axis from an adult sperm whale off Andenes (B. Møhl et al., 2003). Sampling rate is $96 \mathrm{kHz}$. (A) Waveform, apparent source evel in $\mu \mathrm{Pa}$; (B) the received power spectral density by averaged periodogram, continuously on 32-sample windows, Hamming weighted; (C) continuous spectrogram, Hanning weighted, calculated on 128 pts-zero-padded FFT windows of 32 samples; (D) click scalogram by Meyer continuous wavelet transform envelope. (C) and (D) greyscales span 180-230 dB re $1 \mu \mathrm{Pa} 2 / \mathrm{Hz}$, apparent source level.

Temporal patterns of click series

Sperm whale clicks were also chosen as a possible source for this work for the known steadiness of the click production rates. The obvious advantage is the possibility for the monitoring system to search the environment for steady and coherent responses, as a means of raising the detection thresholds and, as a result, reducing false alarm rates. Sperm whale clicks are mostly sequential and interclick-intervals (ICIs) rarely exceed $5 \mathrm{~s}$. Most commonly encountered are the so-called 'usual clicks', which are produced a few seconds after the feeding dive starts and end a few minutes before surfacing. ICIs of usual clicks span 0.5 to 2 s. Clicks of ICI lower than $0.1 \mathrm{~s}$ are called rapid clicks, and those of ICI higher than a few seconds are called slow clicks. Creaks are series of clicks with a much higher repetition rate, as high as $200 \mathrm{~s}-1$, and are believed to be used for sonar and foraging exclusively. Sperm whales are also known to produce 'codas', defined as short sequences (1-2 s) of clicks of irregular but geographically stereotyped ICIs (Pavan et al., 2000; van der Schaar \& André, 
2006). A more elaborate form of ICI analysis performed on usual clicks showed that the ICI may follow a rhythmic pattern that could be used as a signature by individuals of the same group. This pattern is a frequency modulation of the click repetition rate of usual clicks (André \& Kamminga, 2000).

\section{Ambient noise imaging to track non-vocalising sperm whales}

Sound propagates in water better than any other form of energ, thus cetaceans have adapted and evolved integrating sound in many vital functions such as feeding, communicating and sensing their environment. In areas where marine mammal monitoring is a concern, detection and localization can therefore be efficiently achieved by passive sonar, but provided that the whales are acoustically active. When near or at the surface, where they may remain for 9 to $15 \mathrm{~min}$ between dives (André, 1997), sperm whales (Physeter macrocephalus) are known to stop vocalizing (Jaquet et al., 2001). Not discarding the possibility of deploying static active sonar solutions that would scan the high-risk areas, the concern that whales are highly sensitive to anthropogenic sound sources (Richardson et al., 1995) has motivated the search for alternative passive means to localize them. The whale anti-collision system (WACS) is a passive sonar system to be deployed along maritime routes where collisions are a concern for public safety and cetacean species conservation (André et al., 2004a,b; 2005). The WACS will integrate a three-dimensional localization passive array of hydrophones and a communication system to inform ships, in real-time, of the presence of cetaceans on their route. To detect silent whales, alternatives to conventional passive methods should be explored in order to avoid or complement active sonar support. In the present case, i.e. a group of sperm whales consisting of silent and vocal individuals, using the latter's highly energetic clicks might prove effective as illuminating sources to detect silently surfacing whales. Ambient noise imaging (ANI) uses underwater sound just as terrestrial life forms use daylight to visually sense their environment. Instead of filtering the surrounding ocean background noise, ANI uses it as the illuminating source and searches the environment for a contrast created by an object underwater (Potter et al., 1994; Buckingham et al., 1996). Although ANI is fraught with technical difficulties and has been validated, to date, at relatively short ranges, it opens new insights into acoustic monitoring solutions that are neither passive nor active in the strict sense. The solution introduced in this paper is conceptually based on both ANI and multi-static active solutions, where the active sources are produced by surrounding foraging sperm whales at greater depths (from $200 \mathrm{~m}$ downwards), which vocalize on their way down and at foraging depths (Zimmer et al., 2003), and in reported cases, likely on their way up until a few minutes before surfacing (Jaquet et al., 2001). The full analysis can be found in Delory et al., 2007.

A comparable approach was introduced for the humpback whale (Megaptera novaeangliae) off eastern Australia (Makris \& Cato, 1994; Makris et al., 1999). In this study, if the solution were to be applied for monitoring purposes, it would be difficult to implement due to the need for near real-time shallow water propagation modelling as humpback whale vocalizations' spectra peaks are at rather low frequencies and as a result happen to be severely altered in the shallow water waveguide. This may prevent correct pattern matching between the direct and reflected signals unless accurate modelling techniques are applied. Comparatively, sperm whales' vocalizations spectra are considerably wider, higher in frequency, and of greater intensity. Their transient nature also makes received signals less prone to overlaps. Furthermore, our interest is in the propagation of these clicks in deep 
water and at relatively shorter distances, where the wave propagation problem is more tractable than for shallow water and long distances. These differing characteristics motivated us to revisit this passive approach and test the efficiency of using deep diving sperm whale clicks as a source to illuminate silent whales near the surface. Amongst numerous constraints, a prerequisite for sperm whale clicks to be used as active sources is that acoustically active whales should be close and numerous enough to create a repeated detectable echo from silent whales. The chorus created by these active whales should occur day and night and possibly all year long. Hence the following demonstration relies on the condition that whales are foraging in a group spread over not more than a few Squire kilometres and where a substantial amount of them are present within that range. Such a scenario has been observed consistently in the Canary Islands (André, 1997) and in the South Pacific (Jaquet et al, 2001), where sperm whales tend to travel and forage in groups of around ten adults, mostly female, spread over several kilometre distances with a separation on the order of one kilometre between individuals. In addition to the above, a substantial amount of information on temporal, spectral and directional aspects of the sources is essential (see section 2).

The essential information is that we can rely upon a high click repetition rate that may generate better estimates in a short time period. We believe that simulations that would implement all known types of click temporal patterns would probably not add significant information at this phase of the study. Consequently, our demonstration will contemplate usual clicks only. As a result, in a simulation where a given group of sperm whales are clicking in chorus, each individual will be assigned an ICI sampled from a uniform probability density function on the [0.5;2] second interval.

In order to evaluate the possibility of detecting and localizing silent whales near the surface using other conspecifics' acoustic energy, information on sperm whale acoustics was analysed and computed to create a simulation framework that could recreate a real-world scenario. Amongst other modules, a piston model for the generation of clicks is described that accounts for the data available to date (Delory et al, 2007). The modelled beam pattern supports the assumption that sperm whale clicks may be good candidates as background active sources. A sperm whale target strength (TS) model is also introduced that interpolates the sparse data available for large whales in the literature.

\section{$3 D$ simulation of sperm whale wave sound}

3D simulation of wave propagation from source-to-receiver and source-to-object-to-receiver in the bounded medium is implemented by software that we designed based on a raytracing model. This well documented and thoroughly utilised method provides good approximation of the full wave equation solution when the wavelength is small compared to water depth and bathymetric features. As seen above, whale TS and click spectra curves prompted our approach only for frequencies above $1 \mathrm{kHz}$, i.e. a $1.5 \mathrm{~m}$ wavelength, a value far smaller than any other physical scale in the problem.

\section{Bathymetry and sound speed profile}

Bathymetric data between the islands of Gran Canaria and Tenerife (Canary Islands, Spain) were obtained with a SIMRAD EM12 multibeam echo-sounder and provided by S. Krastel, University of Bremen, Germany. The bathymetric map horizontal resolution is $87 \mathrm{~m}$. Sound speed profile was estimated by salinity, temperature and pressure measurements up to 1000 $\mathrm{m}$ applied to Mackenzie's equation, and from $1000 \mathrm{~m}$ to the ocean bottom ( $>3000 \mathrm{~m}$ at many 
locations) by linear extrapolation and increasing pressure, while considering temperature and salinity constant, because no deeper data were available to us. The resulting profile was close to typical North Atlantic sound speed profiles found in the literature.

\section{Boundaries}

The operating mechanisms at the surface and seafloor boundaries were incorporated through their physical characteristics. Sea surface effects were limited to reflection loss, reflection angle and spectral filtering. Surface reflection loss was estimated by the Rayleigh parameter, as a function of the acoustic wavelength and the root-meansquare amplitude of surface waves. Angles of reflection were determined by the Snell law, whereas neither surface nor bottom scattering were modelled. Sea-floor effects were limited to reflection loss and reflection angle.

\section{Other parameters}

An arbitrary number of acoustically active whales and one passive object defined by a 3D TS function were arbitrarily positioned in the three dimensions. All active whales were assigned a different and arbitrary waveform, the spectral information of which was estimated and affected the absorption parameter as well as the source radiation pattern. To test the efficiency of arbitrary hydrophone arrays, beamforming was processed at the receiver location by mapping direction of arrival into phase delays and recreating the sound mixture at all sensors. To ease the implementation and testing of the ray solution, a graphical user interface was created under Matlab and called Songlines.

\section{Implementation}

We first delimited a $5 \mathrm{~km} \times 5 \mathrm{~km}$ square area around the monitoring point, located at $40 \mathrm{~m}$ depth, half-way between Tenerife and Gran Canaria islands (Canary Islands, Spain), where 8 clicking whales of $10 \mathrm{~m}$ size are pseudo-randomly positioned between a depth of $200 \mathrm{~m}$ and $2000 \mathrm{~m}$, with the condition that animals maintained a minimum distance of $1 \mathrm{~km}$ between each other. One silent whale was at $100 \mathrm{~m}$ depth and at a controlled distance from the monitoring point of $1000 \mathrm{~m}$. All whales travel in the same direction at a 2-knot horizontal speed and random elevation. Inter-click intervals, radiation patterns and maximum intensities were set according to the above sections. The simulation setup described above was run 200 times with all active whales randomly repositioned with 1000 $\mathrm{m}$ minimal inter-individual separation and the silent whale being $1000 \mathrm{~m}$ away from the buoy. This amounted to a total of 1600 simulations, each calculating the resulting signals at the buoy stemming from one vocal and one silent whale. For each click produced in a simulation the following information was stored: whale position (vocal and silent), on-axis click sound pressure level, piston model diameter, environmental conditions (wave height, reflection ratio at the bottom, ambient noise level and type), ray angular tolerance, azimuth and elevation of the whale, levels, bearings and delays of the reverberated clicks arriving at the buoy. Every click produced by a single whale created 12 paths of measurable arrival levels at the buoy (see Figure 2): three from its source to the buoy (direct, surface- and bottom-reflected); three to the silent whale, each producing another three paths to the buoy. Consequently, the signal at the buoy was altered 9 times by the silent whale.

\section{Results}

Figure 3 shows the distribution of the received levels at the buoy from rays reflected by the silent whale. The number of echoes represents those received out of the 72 reflected rays ( 8 


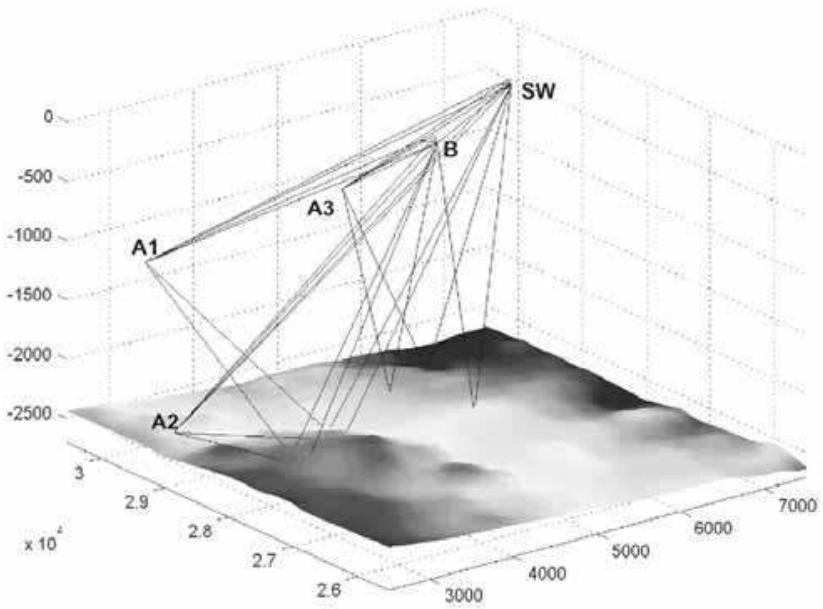

Fig. 2. 3D representation of rays with bottom, surface and object reflections with varying bathymetry resulting from our simulation software Songlines. A1-3, 3 vocal whales; SW, silent whale at $100 \mathrm{~m}$ depth; B, monitoring buoy, here located half-way between Gran Canaria and Tenerife Island $(\mathrm{km} \mathrm{28})$ on the maritime channel. Ray paths account for vocal whale to buoy, vocal whale to non-vocal whale, silent whale to buoy, and their respective bottom and surface reflection paths. All dimensions are in metres.

clicks create 3 paths to the silent whale, each resulting in another 3 paths to the buoy) for each scenario. Signal level distribution is centred on sea-state 1 background noise level (1-30 $\mathrm{kHz}$ ) with a right-hand side tail decreasing until seastate 3 background noise level. As seastates are rarely below 2, especially in the Canary Islands, a first conclusion is that techniques to increase the SNR must be applied to ensure reasonable detection rates. These techniques could build upon the following observations:

1. The fact that clicks are to be repeated on an average of 1 click per second and per whale, implies that the silent whale is likely to be illuminated at least at this rate, and in the rather conservative case that only one whale is a contributing source. Integrated on a 10 $\mathrm{s}$ window, the coherent addition of the silent responses is to increase the SNR by at least $10 \mathrm{~dB}$.

2. A beam-formed phased array would increase the SNR, with the additional benefit of resolving bearing information of the silent whale. Moreover, the broadband nature of the signals of interest here permits the use of sparse arrays of high directionality because frequency-specific grating lobes do not add up coherently in space. This technical scenario was simulated with Songlines. A 4 m-diameter ring array of 32 omnidirectional hydrophones was beam-formed in the time-domain on one typical scenario, under the same control parameters as above. The silent whale was positioned $100 \mathrm{~m}$ deep and $1500 \mathrm{~m}$ away from the antenna. The software also allowed recreating the full waveforms resulting from the multi-path propagation of clicks to the buoy. Each whale produced a click at a random ICI taken from a uniform distribution in the 0.5-1 s interval during a $25 \mathrm{~s}$ period. Whales were separated by at least $1 \mathrm{~km}$ and repositioned every $5 \mathrm{~s}$ according to a group horizontal speed of 2 knots. The rest of the simulation settings remained unchanged. Results are presented in Figure 3. 

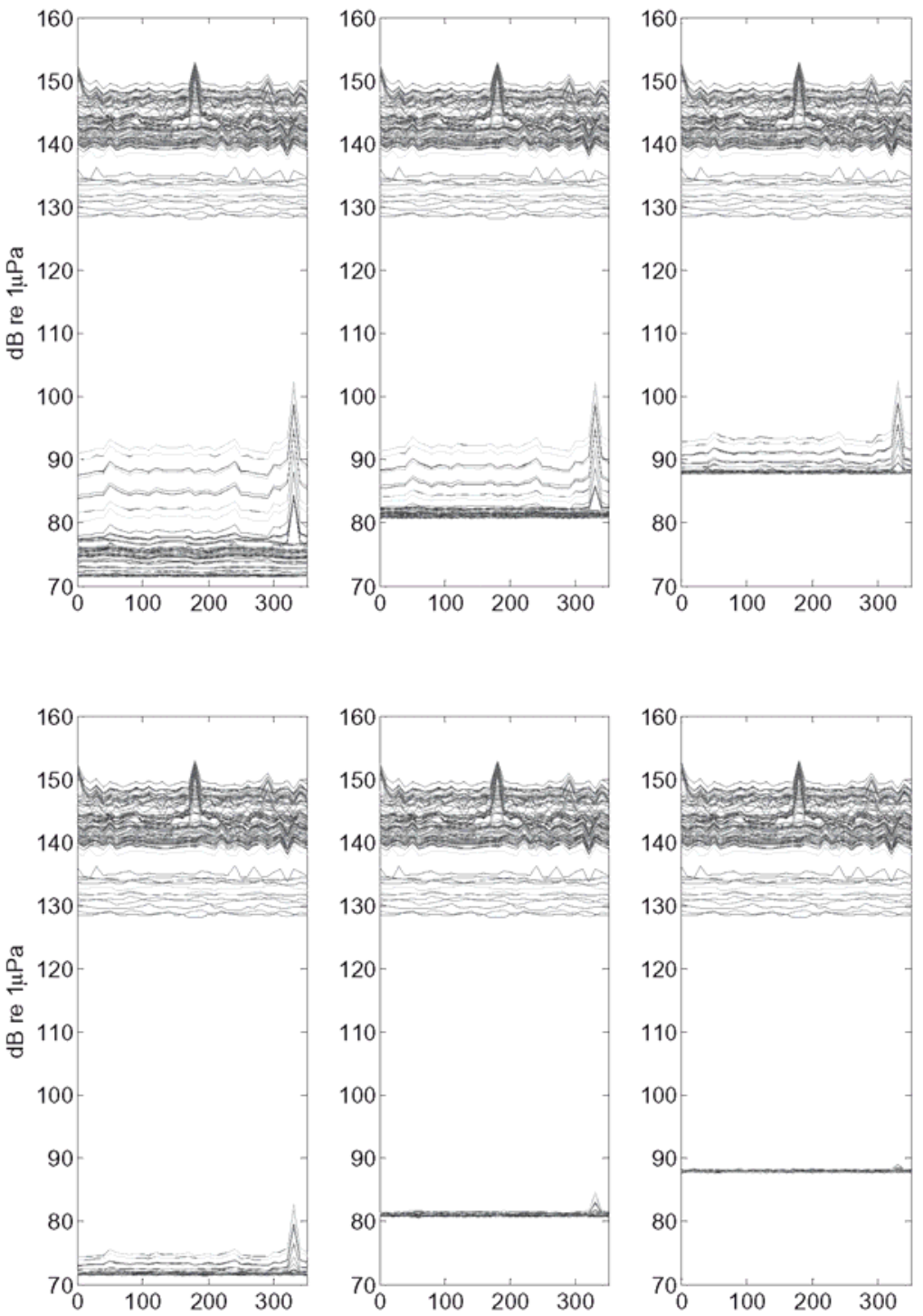

Fig. 3. Received levels on the 32 time-based beam-formed beams of a $\varnothing 4 \mathrm{~m}-32$-sensorantenna for sea state 1, 3 and 6 (left to right) and three passive-active whale types of orientation: from top to bottom: whale angle of view is near beam aspect, and tail-aspect (see text). Array DI is $12 \mathrm{~dB}$ (see text). The simulated silent whale is at $330^{\circ}$ azimuth, $100 \mathrm{~m}$ depth, $1100 \mathrm{~m}$ horizontal distance from the buoy. The cumulated plot results from a 25-s period with 8 whales clicking at depth (see text). Total number of clicks was 189. Beams are altered by the direct and reverberated paths from the vocal whales' clicks directly to the buoy (90 $\mathrm{dB}$ and over). 
3. Matched filtering using pre-localized sources could raise the SNR in cases when seastate and the resulting greater noise levels and reverberations alter the detection rates. However, as clicks are highly directional, matched filtering in the case of sperm whales may not always perform as expected as both source signal and reverberated replicas tend to differ when the source heading changes. As seen in the previous section on click time-frequency characteristics, both time and frequency contents are angle-dependent. As this angle is random to the receiver in most cases, the hypothesis of a deterministic signal is not fulfilled and thus matched filtering would not be optimal. It is also likely that matched filtering would be less efficient at greater ranges, where signals are more distorted. According to Daziens (2004), sperm whale clicks matched filtering was indeed outperformed by an energy detector for ranges greater than $3000 \mathrm{~m}$. In fact, the latter outperformed matched filtering only for sperm whale click detection. Detection ranges were then nearly doubled as compared to matched filtering, for the same source level, detection and false-alarm probabilities, of $50 \%$ and $1 \%$ respectively. In our case, as the two-way propagation (source to silent whale to receiver) results in greater attenuation and distortion than those resulting from a one-way propagation of the same distance, it is expected that the energy detector will outperform matched filtering.

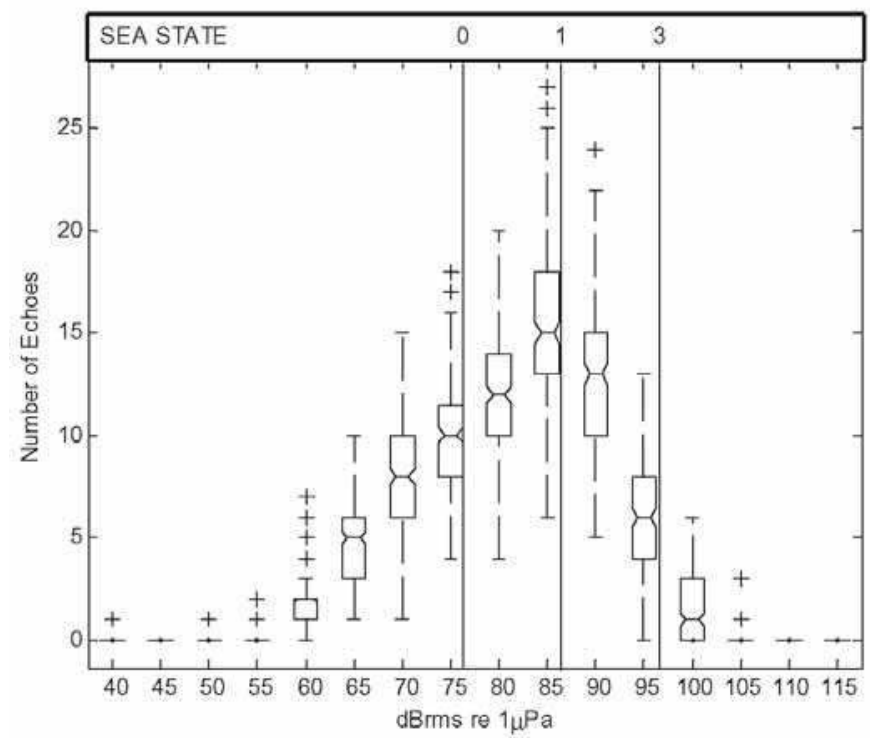

Fig. 4. Statistical plot of the simulated received RMS levels of clicks reflected on a silent whale located at $1000 \mathrm{~m}$ distance from the buoy (see text for details on simulation settings). Ordinates represent the median number of contributing clicks per simulation drawn from 200 simulations (each simulation includes 8 vocal whales clicking once). Also plotted are lines at the lower quartile and upper quartile values. The whiskers are lines extending from each end of the box to show the extent of the rest of the data. Outliers are data with values beyond the ends of the whiskers. Notches over and below median values are medians' 95\% confidence intervals. Sea-states 0 to 3 and above noise levels in the 1-30 kHz bandwidth are represented (calculated from Urick, 1996). 
4. In view of the above, which advises a simplistic preprocessing method based on beamforming and signal energy, we plotted the received signal intensity distributions from 25 ms time-intervals in Figure 4 (no background noise, no beam-forming) and Figure 5 (with background noise and beam-forming). Figure 4 shows that the resulting probability density function is bimodal, where the low-level mode represents the click energy reverberated from the silent whale, and the high-level mode, centred above 120 $\mathrm{dB}$, stems from the click direct, surface and bottom reflected energy at the receiver. We anticipate that simultaneous occurrence of these two modes on a limited number of beams could prove robust for a decision stage.
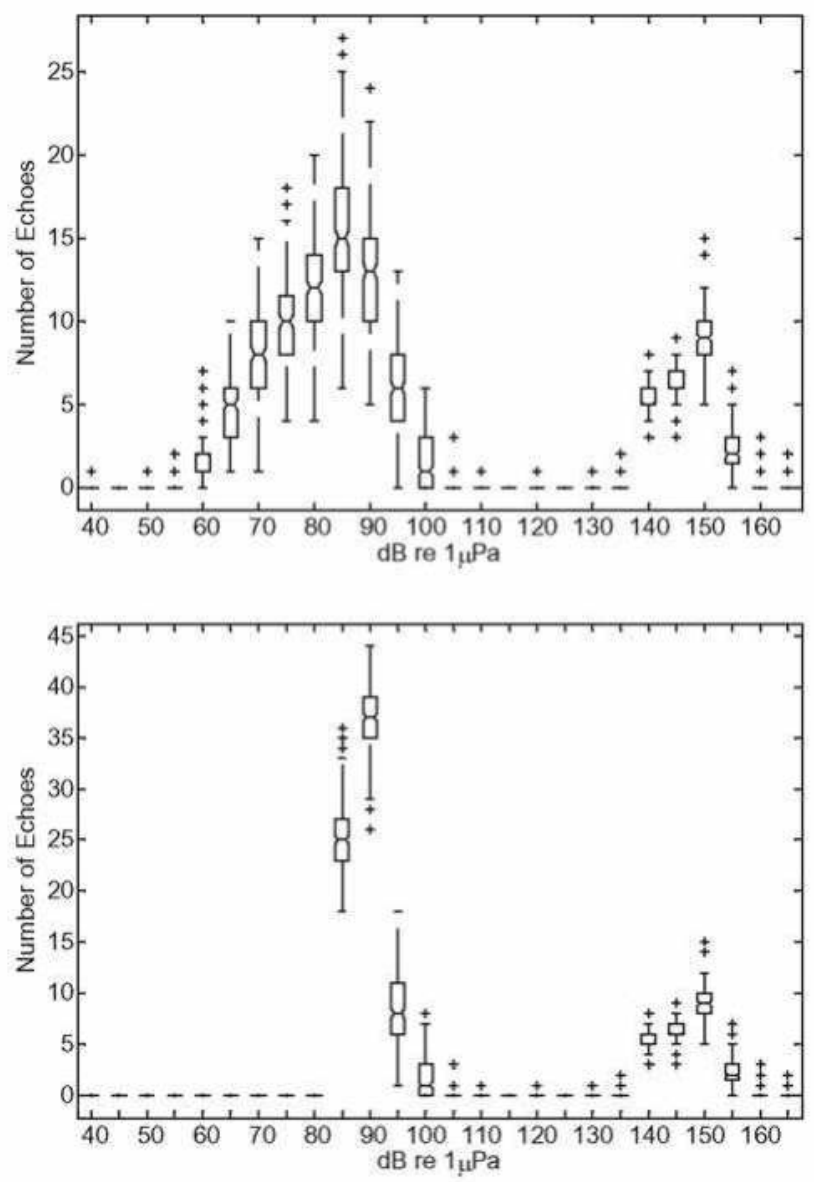

Fig. 5. Distribution of direct, surface, bottom-reflected and silent-whale reverberated clicks. The top figure is the level-expanded version of Figure 4, which highlights the bimodal aspect of the received level distribution. The bottom figure represents the resulting distribution at sea-state 1 with an omni-directional receiver. The same results are obtained on one beam for sea-state 3 after beam-forming with the antenna described in the text. 


\section{Space-time and hybrid algorithms for the passive acoustic localisation of sperm whales and vessels}

The prominent approach, described in the previous section, for the passive acoustic localisation of cetaceans is based on the estimation and spatial inversion of time differences of arrival of an emitted signal at spatially dispersed sensors, which form an array. A second class of methods, space-time methods, originated from underwater applications such as sonar and found valuable applications in other fields such as the analysis of seismic waves or digital communications. In the latter, a significant amount of research has been devoted to space-time methods leading to powerful developments over the last 20 years. This approach has indeed shown to provide more accurate results than TDOA-based methods (Krim \& Viberg, 1996). By maximising the mutual information between the source signal and array out- put, space-time methods achieve reduced variance in position estimates. Furthermore they offer simple means for the localisation of multiple simultaneously radiating sources. While the case of narrowband signals is well documented, the application of space-time methods to broadband signals, such as those emitted by sperm whales, only recently found satisfying developments in terms of complexity and accuracy (Dmochowski et al., 2007). These broadband developments could be imported and largely benefit the localisation of cetaceans: they indeed outperform TDOA-based methods even with a similar small number of sensors, a performance, which increases in harsher conditions with high levels of noise and reverberation. It is not the intention of this paper to thoroughly compare TDOA-based and space-time methods: this is an evaluation, which requires fairness and constant updates. Rather, this paper aims to illustrate the interest of developing an alternative frame concerning localisation, which may be well suited for certain array configurations. It will present the newly developed and challenging principles behind these methods and the results they can achieve for the passive acoustic localisation of multiple sperm whales and vessels. The principles which underlie the increased robustness of spacetime methods will be recalled, and remarks are made concerning other interesting results which can be obtained via these methods such as broadband beam pattern estimation and dynamic estimation of attenuation factors. The full description of the approach can be found at Houégnigan et al., 2010.

A promising new class of hybrid localisers is introduced and its abilities for the localisation of sperm whales are shown. An important achievement of these hybrid localisers, in the case of compact arrays, is the reduction of the necessary processing time for results equivalent to those obtained for space-time methods. All of the developments to follow are intended to be included in a real-time developed at the Laboratory of Applied Bioacoustics (LAB) of the Technical University of Catalonia, for the passive monitoring of cetaceans from deep-sea observatories (http:/ / listentothedeep.com).

\subsection{General frame of the technical developments}

\section{Propagation Model}

In this paper a compact array and real far-field sources are under consideration, far beyond the Rayleigh limit (Ziomek, 1995). The main focus is on the quality of bearing estimation provided by space-time methods and hybrid methods rather than on their range estimation capabilities, even though high-resolution space-time estimates of range could be obtained under certain conditions (Dmochowski et al., 2007). The model moreover focuses on 
broadband sound, hence throughout this paper when reference is made to "cetaceans" this actually only refers to cetaceans producing broadband sound; note that the developments are valid for all types of broadband sounds, which includes some vessel sounds.

A three-dimensional array of $\mathrm{M}$ sensors is assumed. Due to propagation, each sensor receives attenuated, phased and noisy versions of the signal $s$ emitted by a cetacean at spherical position $\underline{\mathbf{r}}_{\mathrm{s}}=\left[\mathrm{r}_{\mathrm{s}} \Theta_{\mathrm{s}} \Phi \mathrm{s}\right]$. The coordinates of $\underline{\mathbf{r}}_{\mathrm{s}}$ respectively represent range, azimuth and elevation.

The signal $x_{i}(t)$ received at the $i^{\text {th }}$ sensor at instant $t$ is modelled as:

$$
x_{i}(t)=\alpha_{i}\left(\underline{r}_{s}\right) \cdot s\left(t-\tau_{j, i}\left(\underline{r}_{s}\right)\right)+v_{i}(t),
$$

where $v_{i}$ represents the additive noise at sensor $i$, which may include background and propagation noise, reverberation, and electronic noise. If sensor $j$ is taken as the reference sensor, the $i^{\text {th }}$ signal can be expressed by using the propagation delay $\tau_{j, i}\left(\underline{r}_{s}\right)$ which is related to the path difference between the signals received at sensors $j$ and $i$. Each $x_{i}$ is thus modelled as a noise-corrupted phased and attenuated by distance (term $\left.\alpha_{i}\left(\underline{r}_{s}\right)\right)$ and version of the signal $s$ emitted by the cetacean or broadband sound source.

\subsection{Methods for the localisation of cetaceans}

\section{Methods based on Time Differences of Arrival (TDOA)}

To understand the hybrid methods presented below, it is necessary to understand some aspects of TDOA-based methods (see section 3), but also to compare them to space-time methods.

The basic principle behind TDOA-based methods is that the time differences of arrival between the signals received at each sensor are related to the propagation path and the position of the estimated source. Hence TDOA-based methods feature two main steps: firstly time-delay estimation (TDE), and secondly a time-space inversion which consists in forming the position of the radiating source from the group of estimated TDOA related to the array geometry.

\section{Limits of TDOA-based methods}

The estimated time-delays between two noisy signals are themselves corrupted with broadband noise. Generalised Cross Correlation can improve estimation but this may not be sufficient. Each of the noisy estimates is then used in a time-space inversion phase and participates in the construction of a location estimate strongly affected by noise. This is a severe a priori hindrance that causes anomalies and high variance in the localisation results even if sophisticated statistical post-processing is applied. Combining all the sensors at disposal and not using only pairs could yield a strong noise reduction: space-time and hybrid methods precisely carry out such a beneficial processing. Indeed, the distinction between the spatial propagation of the signal emitted by cetaceans as opposed to the supposedly incoherent nature of noise offers powerful means of spatial separation.

Space-time methods

Several space-time methods were implemented for the localisation of cetaceans. The spacetime terminology covers beamformers, spatial spectral estimators, and more generally methods based on the processing of a spatial observation vector estimated at various time 
instants. Space-time methods construct a spatial spectrum by virtually steering the array in various directions and estimating the received power (in some cases only a power-like index is estimated). When steered in the direction of a source the power received by the array and the signal-to-noise ratio will be maximised, and hence the spectrum will exhibit a high peak, whereas in directions where no sound or only low-power incoherent noise is radiated the received power will be weak and therefore the spatial spectrum will be relatively flat. Another way to interpret space-time methods and in particular spatial spectral estimators is to link them to frequency estimation; indeed these methods do extract information concerning a spatial frequency: the wavenumber. There exists a strong theoretical link between spatial frequency estimation and the more familiar temporal frequency estimation to the point that many methods moved from one domain to the other over the last decades (Johnson, 1982).

Power estimation

A power $P\left(\theta_{k}, \phi_{k}\right)$ is received when the array is steered in the direction $\left(\theta_{k}, \phi_{k}\right)$. Steering is concretely achieved by delaying each signal according to the theoretical delays observed at each sensor for a waveform coming from direction $\left(\theta_{k}, \phi_{k}\right)$. One sensor is to be chosen as reference.

Hence when only one source is present its estimated bearing $\left(\hat{\theta}_{S}, \widehat{\phi}_{S}\right)$ is given by:

$$
\left\langle\hat{\theta}_{S}, \hat{\phi}_{S}\right\rangle=\underset{k}{\operatorname{argmax}}\left(P\left(\theta_{k}, \phi_{k}\right)\right)
$$

Multiple sources can be located by searching for multiple peaks in the spatial spectrum. The accuracy and resolution of the spatial spectrum is related to the way the calculation of power is carried out. In this paper, the general frame for power calculation is based on the estimation of a spatial correlation matrix and on various spatial estimators, which function as spatial filters.

Derivation of the spatial correlation matrix

The spatial correlation matrix (SCM) carries information about the correlation between the signals received at the sensors and the phase and amplitude differences between them. Other names may be encountered in literature such as space-time covariance matrix, spatiospectral correlation matrix or spectro-temporal covariance matrix, but the same spatial second order statistics is always meant.

The SCM noted as $\Re$ is defined by:

$$
\mathfrak{R}=E\left\{x x^{H}\right\}
$$

where $E\{\}$ denotes mathematical expectation and where $H$ indicates Hermitian conjugation.

In practice the signals' finite nature only permits an estimation of $\mathfrak{R}$. Estimation is made more difficult by short duration signals like some of those emitted by cetaceans. In a discrete frame, the most widely used estimate of $\widehat{R}$ can be expressed as:

$$
\widehat{\Re}=\frac{1}{N_{S}} \sum_{n=1}^{N_{S}} z_{n} z_{n}{ }^{H},
$$


where $N_{S}$ is the number of samples corresponding to the signal, where $z_{n}$ is a spatial observation vector at instant $n$.

$\widehat{R}$ should not be confused with the cross-correlation function $R_{x_{i} x_{j}}$ as presented in section (2.1.2), this will be important for the hybrid methods presented in 2.3.

At instant $\mathrm{n}$, i.e. for the $\mathrm{n}^{\text {th }}$ sample acquired by the array, the observation vector is given by $z_{n}=\left[\begin{array}{lllll}x_{1}(n) & x_{2}(n) & \ldots & x_{M}(n)\end{array}\right]^{\mathbf{T}},(2.5)$.

Derivation of the steered spatial correlation matrix

The steered spatial correlation matrix $\mathfrak{R}\left(\theta_{k}, \phi_{k}\right)$ is the spatial correlation matrix associated with the array when it is virtually steered in the direction $\left(\theta_{k}, \phi_{k}\right)$ to estimate the power received by the array from that particular direction. Steering in the direction $\left(\theta_{k}, \phi_{k}\right)$ is done by adequately delaying the received signals with regard to a chosen reference sensor. The observation vector $z_{n}$ then transforms to $z_{n}^{(k)}$ and $\widehat{R}\left(\theta_{k}, \phi_{k}\right)$ can then be expressed as :

$$
\widehat{\Re}\left(\theta_{k}, \phi_{k}\right)=\frac{1}{N_{S}} \sum_{n=1}^{N_{S}} z_{n}^{(k)} z_{n}^{(k) H}
$$

For example if the $\mathrm{j}^{\text {th }}$ sensor is chosen as a reference, the expression of $z_{n}^{(k)}$ is given by:

$$
z_{n}^{(k)}=\left[x_{1}\left(n-\delta_{j 1}^{(k)}\right) \quad x_{2}\left(n-\delta_{j 2}^{(k)}\right) \ldots x_{M}\left(n-\delta_{j M}^{(k)}\right)\right]^{\mathbf{T}},
$$

where $\delta_{j m}^{(k)}$ represents the theoretical delay in samples between the signals at the $\mathrm{j}^{\text {th }}$ and $\mathrm{m}^{\text {th }}$ sensor for a far field source radiating from direction $\left(\theta_{k}, \phi_{k}\right)$. Note that this process may suffer slight limitations from the sampling frequency since the computable delay in samples and the actual delay for direction $\left(\theta_{k}, \phi_{k}\right)$ do not exactly match.

\begin{tabular}{|l|l|c|c|}
\hline $\begin{array}{l}\text { Spal Spectral } \\
\text { Estimator }\end{array}$ & Power estimate & $\begin{array}{c}\text { Theoretical } \\
\text { Spectral } \\
\text { resolution and } \\
\text { accuracy }\end{array}$ & $\begin{array}{c}\text { Computation } \\
\text { time }\end{array}$ \\
\hline $\begin{array}{l}\text { Steered Response } \\
\text { Power (SRP or } \\
\text { Bartlett) }\end{array}$ & $P\left(\theta_{k}, \phi_{k}\right)=w^{T} \cdot \hat{R}\left(\theta_{k}, \phi_{k}\right) \cdot w$ & + & + \\
\hline $\begin{array}{l}\text { Capon } \\
\text { (Minimum Variance) } \\
{[15]}\end{array}$ & $P\left(\theta_{k}, \phi_{k}\right)=\frac{1}{w^{T} \cdot\left(\hat{R}\left(\theta_{k}, \phi_{k}\right)\right)^{-1} \cdot w}$ & ++ & ++ \\
\hline $\begin{array}{l}\text { Eigenvalue } \\
\text { decomposition (EIG) }\end{array}$ & $P\left(\theta_{k}, \phi_{k}\right)=\lambda_{\max }\left(\theta_{k}, \phi_{k}\right)$ & +++ \\
\hline $\begin{array}{l}\text { MuSiC } \\
{[14]}\end{array}$ & $P\left(\theta_{k}, \phi_{k}\right)=\frac{1}{w^{T} \cdot \hat{\Pi}_{\left(\theta_{k}, \phi_{k}\right)} \cdot w}$ & ++++ & +++ \\
\hline $\begin{array}{l}\text { Other estimators: } \\
\text { ESPRIT, Root-MusiC } \\
\text { Propagator...[16] }\end{array}$ & $\ldots$ & $\ldots$ & ++++ \\
\hline
\end{tabular}

Table 1. Description of a few spatial spectral estimators 
where $w=\left[1^{1} \ldots 1^{m} \ldots 1^{M}\right], \lambda_{\max }\left(\theta_{k}, \phi_{k}\right)$ denotes the maximum eigenvalue of $\hat{R}\left(\theta_{k}, \phi_{k}\right)$, and $\widehat{\Pi}_{\left(\theta_{k}, \phi_{k}\right)}$ denotes the noise subspace of $\widehat{\Re}\left(\theta_{k}, \phi_{k}\right)$.

Based on the matrix defined in (2.6) we present in table 1 various spatial spectral estimators used to obtain our results (see below). EIG, Capon, and MuSiC are often referred to as highresolution algorithms, and MuSiC is also labelled as subspace-based.

\section{Hybrid spatial spectral estimation}

The newly defined and developed hybrid methods are composed of three steps related both to space-time methods and TDOA-based methods.

\section{Step 1: Calculation of the generalised cross-correlation for all pairs of sensors}

Note that using other functions than GCC at this step may bring other interesting results.

Step 2: Construction of a Steered hybrid SCM $\widehat{\Re}_{h y b}\left(\theta_{k}, \phi_{k}\right)$ based on the generalised crosscorrelation functions.

There exists a clear mathematical relationship between the cross correlation and the hybrid SCM such that the element $\widehat{r}_{i j}$ on the ith line and jth column of $\widehat{R}_{h y b}\left(\theta_{k}, \phi_{k}\right)$ is given by:

$$
\widehat{r}_{i j}=\widehat{R}_{x_{i} x_{j}}\left(\delta_{i j}^{(k)}\right),
$$

$\hat{R}_{x_{i} x_{j}}$ represents the estimated generalised cross-correlation function between the signals at the $\mathrm{i}^{\text {th }}$ and $\mathrm{j}^{\text {th }}$ sensor. The use of $\delta_{i j}^{(k)}$ follows from Eq. (2.7). The operation in Eq (2.8) selects realisable delays within the cross-correlation functions and repositions the temporal secondorder statistics in a spatial frame.

Step 3: Space-time power estimation

Space-time power estimation can be conducted based on the steered hybrid covariance matrix $\widehat{\Re}_{h y b}\left(\theta_{k}, \phi_{k}\right)$. The power estimators presented in table (2.2) can be re-used simply by replacing $\widehat{R}\left(\theta_{k}, \phi_{k}\right)$ by $\widehat{\Re}_{\text {hyb }}\left(\theta_{k}, \phi_{k}\right)$.

Nomenclature of hybrid methods

The name of a hybrid method will be composed of two parts: firstly the type of spatial power estimator used and secondly the type of GCC filter used. For example, SRP-SCOT corresponds to a SCOT filter applied to the Cross-Correlation function at step 1 and a Steered Response Power at step 3. Similarly MuSiC-ROTH corresponds to a ROTH filter applied to the Cross-Correlation function in the first phase and a MuSiC Power Estimation in the third phase. When no filtering is done, a standard Cross-Correlation function is used and the hybrid method is almost equivalent to the corresponding space-time method except that the estimated SCM remains hybrid with regard to its construction. In that case we would write for example SRP-hybrid or MuSiC-hybrid to differentiate them from the classical space-time SRP and MuSiC. In the case presented here hybridisation typically consists in going from a temporal second order statistics to a spatio-temporal second-order statistics.

Note that some methods developed by other authors are very close to the class of hybrid methods. This is notably the case of the SRP-PHAT algorithm developed by Griebel and Brandstein (2001). Developed mostly for conference settings with high reverberation it uses firstly the generalised cross-correlation with a PHAT filter and secondly a steered response 
power approach to localise speakers. However the method is obviously derived in a different manner and its authors class it as TDOA-based (DiBiase et al., 2001). Indeed, it does not rely on steered correlation matrices, which would have permitted to relate the spatial and temporal second order statistics and which would formally place their estimator in the hybrid group. To our knowledge, the first technical equivalent of a hybrid method was presented by Dmochowski et al, in 2007, who introduced the parameterised spatial correlation matrix, a powerful framework which inspired the hybrid steered SCM.

\section{Final methodical remarks}

The space-time and hybrid approaches presented here are well suited for far-field cetacean localisation and in particular for broadband cetacean sound. Typically a relatively small number of widely spaced sensors are featured while some cetaceans emit sound with a proportionately high frequency content, which may yield spatial aliasing. Spatial aliasing is a well known but poorly studied phenomenon caused by the relation between the aperture of the array and the wavelengths present in the signal.

The philosophy behind the methods presented here is, as in most TDOA-based methods, to treat the broadband signals received as truly broadband, and not as an artificial composition of narrowband components. This permits to gain accuracy, to mitigate the effects of spatial aliasing and to reduce processing time. In order to implement this time approach for broadband cetacean sound, a simple time-derived spatial correlation matrix is computed. Sophisticated frequency derivations of the SCM (Wang \& Kaveh, 1985) do exist but they may have difficulties in coping with real-time requirements. Furthermore, given the spatial dimensions of most arrays deployed underwater, the frequency approach is likely to be heavily corrupted by spatial aliasing, which will then affect the accuracy of cetaceans' localisation.

\section{A Short Presentation of the datasets and material}

In the frame of the NEMO collaboration (Neutrino Mediterranean Observatory) for neutrino detection (Riccobene, 2009), more than 2000 hours of multichannel recordings were gathered. An underwater station was installed $25 \mathrm{~km}$ East of the port of Catania (Sicily) at approximately $2000 \mathrm{~m}$ depth. The station was equipped with four hydrophones working in a frequency band, which is sufficiently large (from $36 \mathrm{~Hz}$ to $43 \mathrm{kHz}$ ) for the detection, classification and localisation of vocalising cetaceans. The average distance between the sensors was $2.5 \mathrm{~m}$. Data was acquired at a sampling rate of $96 \mathrm{kHz}$. Vocalising sperm whales were detected with an algorithm for the real-time detection of impulsive sounds, which provided an estimation of the onsets and offsets of the sperm whale clicks (Zaugg et al, 2010).

Information from these datasets was extracted to estimate the beampattern and to perform localisation. The calculations were run under Matlab on a desktop with a $2.8 \mathrm{Ghz}$ Pentium IV with limited memory which explains some relatively high calculation (Houégnigan et al, 2010).

\subsection{Results}

\section{Determination of the beam pattern of the array}

The beam pattern represents the variation of intensity or sound pressure level received as the direction of arrival varies, range being fixed. This is valuable information concerning the capability of the array to localise sources. The beam patterns presented in figures 6.1 and 6.2, 
respectively based on SRP and EIG, demonstrate that the array possesses good spatial separation capabilities with regard to bearing even with only four sensors and is not strongly affected by sidelobes, grating lobes and aliasing. A broadband sperm whale click of average energy was selected from the available data sets as a representative reference source. The traces and maxima, in the beampatterns 6.1 (left) and, even more clearly, in 6.1 (right), are related to the power received by the array. This power is itself related to the path difference between the sensors for a particular angular position of the source. The simplest maxima, yet not the most obvious, occurs at the borders of the spectra when the elevation is at $0^{\circ}$ or $180^{\circ}$, i.e. when the source is pointing towards the array from above or from below. This position minimizes the path difference between three hydrophones (i.e. those with cartesian coordinate $\mathrm{z}=0$ in the tetrahedron) and maximizes the power received by the whole array. Given the regular form of the array (the array is almost tetrahedral in shape but not exactly) it is clear that the power received will be invariant by rotation or by certain movements. This is verified by the six other maxima, which can be found in the pattern. There is a clear symmetry among them due to the choice of an azimuth varying from $360^{\circ}$ and not just $180^{\circ}$. In the same way, traces can be explained by considering the array geometry and how the DOA of the source influences the path difference and power received. The 9 traces observed ( 6 traces appear at constant azimuth and 3 traces oscillate with azimuth in a manner reminiscent of a sine wave) show us that certain positions of the source create invariance of the power received, this power being relatively high. In these cases only the power received between pairs of hydrophones is actually maximized and thus only the path difference between pairs of hydrophones is minimized. There are clearly more ways of maximizing the power received for pairs than for triplets of sensors and this explains the extension of the traces and their number. On the whole, the traces observed are strongly dependent on the array geometry in the sense that they follow all the spatial positions, which maximize the power received (or minimize the path difference) in pairs of sensors. With EIG, spectral lines appear much sharper and spatial regions are much more clearly separated in terms of power than with SRP. For localisation, this implies less ambiguity in the estimation through clearer and narrower peaks.
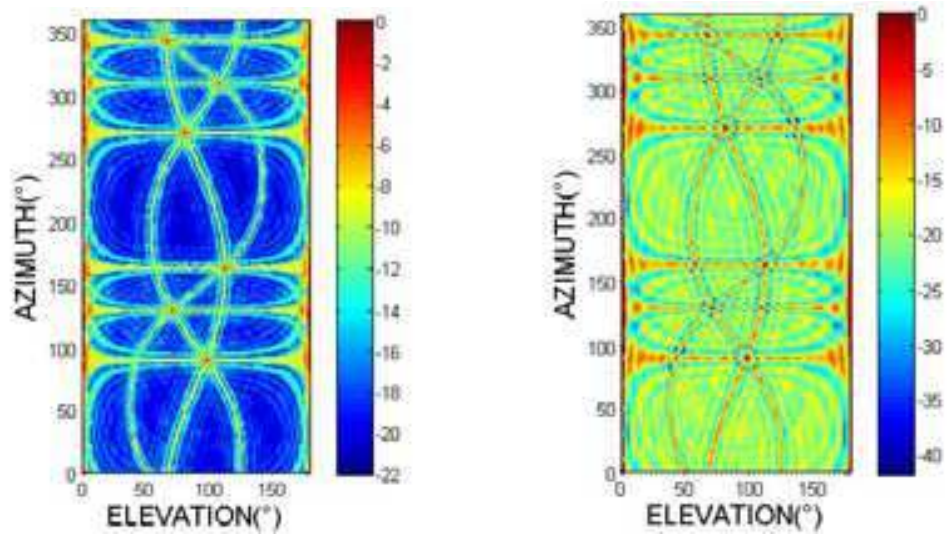

Fig. 6.1. Broadband beam pattern for a broadband click computed through SRP (left); broadband beam pattern for a broadband click, computed through EIG (right). Colour scale indicates average output power in $\mathrm{dB}$. 


\section{Click-by-click localisation}

Click-by-click localisation assumes that each click in a sequence contains information concerning the position of a vocalising sperm whale. Hence applying various spatial spectral estimators to a unique click can give an indication concerning their performance. Among the numerous 5 minutes duration datasets at disposal, the dataset recorded on $14^{\text {th }}$ August 2005 from 3pm to 3.05pm was chosen. In this short sequence 819 impulsive sounds were detected and classified as sperm whale clicks. The localisation procedure was run for the methods presented above. In order to compare the localisation capabilities of those methods a single click of average energy, the $40^{\text {th }}$ in the sequence, was selected. The processing of this click was also used to assess processing time. This will permit to decide on the choice of a suitable algorithm for real-time tracking.

\section{Via Space-time methods}

Figure 6.2 present the spatial distribution of power received for the selected click for spacetime methods. A one-degree resolution was used for the computation of the spectra. There is a clear similarity between them, with spectral lobes, which are characteristic of the array, the strongest of which should converge towards the putative source location. The located source appears without ambiguity as a sharp peak within a dense zone of high power in figures 6.2 (left) and 6.2 (right), respectively for the SRP and EIG algorithms. The spatial spectra for $\mathrm{MuSiC}$ and Capon are not presented here since they provided inconsistent location estimates. The Capon spatial spectrum appeared extremely noisy with many secondary peaks while the MuSiC spectrum was obviously less noisy but did not have a clear unique peak. The circles, which appear in 6.2 and 6.3 are artefacts in the construction of the spatial spectrum. Spectral lines other than circles are actually observed in different positions of the spectrum when the source is at a different position. However, these artefacts are not appearing randomly: in the same way as for the beam pattern, spectral lines appear in correlation with the position of the source and the geometry of the array. This is comparable to frequency estimation where the spacing between the sampling points (sampling rate) constraints the spectrum as much as the spectral content of the signal. Here, the placement of the sensors in the array operates a sampling of space, which has an influence on the spatial spectrum.
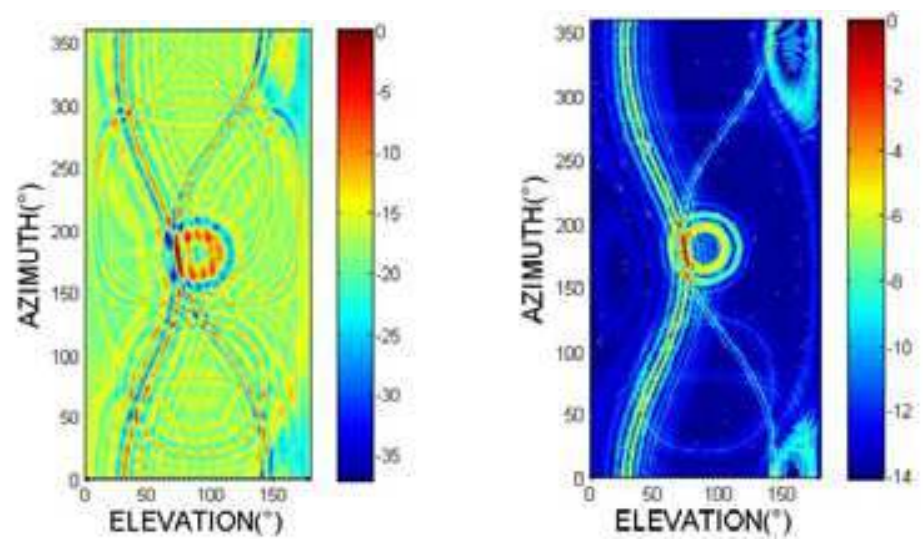

Fig. 6.2. Localisation of a broadband click computed with SRP (left); localisation of a broadband click computed with eigenanalysis spatial spectral estimation (right). Estimated position: $\left(\hat{\theta}_{s}, \widehat{\phi}_{s}\right)=\left\{176^{\circ}, 74^{\circ}\right\}$. Color scale indicates average output power in $\mathrm{dB}$. 
Via Hybrid methods

Figure 6.3 and 6.4 present the spatial distribution of power received for the selected click for the hybrid methods, which were implemented. A one-degree resolution was used for the computation of the spectra. In figure 3.7 a side view of the spatial spectra (corresponding to elevation against power) is shown which permits to evaluate the number of side lobes, the separation between signal and noise for hybrid $\mathrm{MuSiC}$ and to visualise a narrow localisation peak, which is not obvious from 3.6. There is clearly a similarity between the hybrid spectra and the spectra obtained with space-time methods.
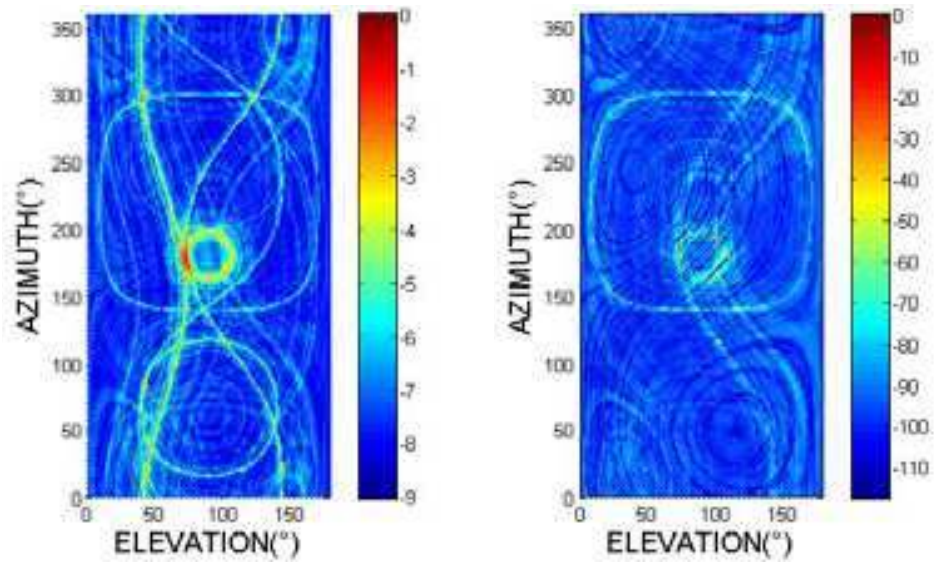

Fig. 6.3. Performance of SRP-ROTH (left); performance of MUSIC-SCOT (right), colour scale indicates average output power in $\mathrm{dB}$.

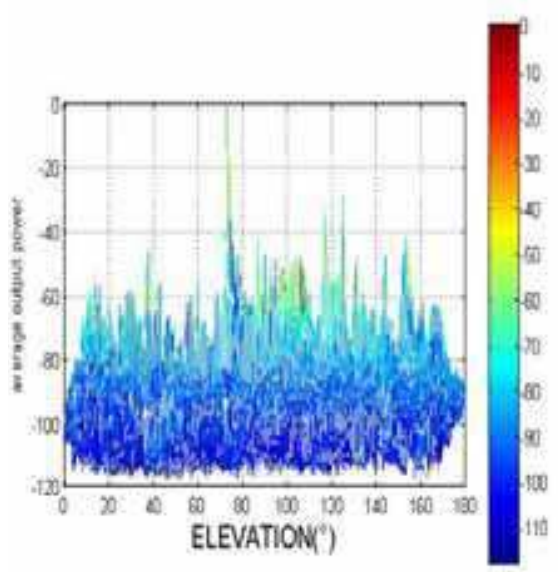

Fig. 6.4. Performance of MUSIC-SCOT, (elevation only), colour scale indicates average output power in $\mathrm{dB}$. 
The located source appears clearly as a sharp peak within the red-coloured zone in figure 6.3, respectively for SRP-ROTH and MuSiC-SCOT. The hybrid EIG algorithm failed to give results, which could compare with its non-hybrid version, it featured large spectral lines of high power which could not correspond to a real scenario and therefore it is not included here. The performance achieved by SRP-ROTH was very similar to that obtained for the non-hybrid EIG, with a reduced processing time (Houégnigan et al, 2010). With SRP-SCOT various high amplitude secondary peaks appeared which was not the case was for SRPROTH.

The Capon and Music methods did seem to perform more reliably when hybridised. They could isolate a main peak, which reduced ambiguity as figure 6.4 shows for MuSiC-SCOT. MuSiC-SCOT and MuSiC-ROTH in particular did achieve a powerful separation of signal (peak) and noise (lower power zones) as could be expected from the (non-hybrid) theory of MuSiC (Schmidt, 1986). The localisation obtained for the hybridised versions of Capon permitted to achieve a consistent localisation but figures are not presented for conciseness. Several secondary peaks appeared for Capon-SCOT but they were not yet problematic; they were not present for Capon-ROTH. In general ROTH hybrids seemed to provide the most reliable localisations.

\section{Tracking of sperm whales and boats}

Repeating the localisation procedure for each of the impulsive sounds detected in a 5minute window allowed to track the movement of emitting sources classified as sperm whales or boats.

\section{Track 1: Dataset 18 ${ }^{\text {th }}$ August 2005, 10 pm}

Besides some isolated locations, which may be anomalies or simply scarcely vocalising sperm whales, two main tracks can be isolated with a clear separation in azimuth and elevation against time. The first one is found close to $\left(\theta_{1}, \varphi_{1}\right)=\left\{160^{\circ}, 60^{\circ}\right\}$ and the second one close to $\left(\theta_{2}, \varphi_{2}\right)=\left\{200^{\circ}, 55^{\circ}\right\}$.

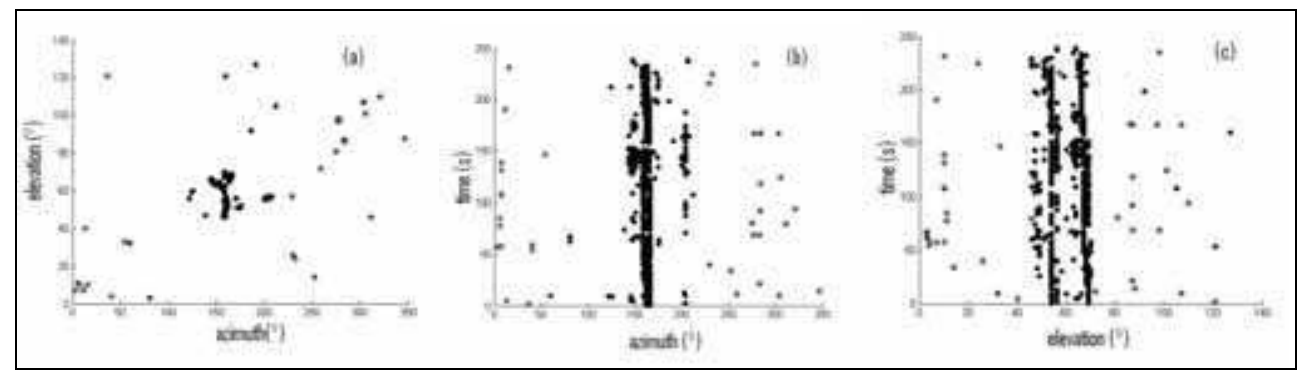

Fig. 6.5. Sperm whale tracking, $18^{\text {th }}$ August 2005, 10pm

\section{Track 2: Dataset 09th August 2005, 09 pm}

776 sperm whale clicks were taken into account for localisation. There are two main clusters of points with sound sources moving around $\left(\theta_{1}, \varphi_{1}\right)=\left\{80^{\circ}, 50^{\circ}\right\}$ and $\left(\theta_{2}, \varphi_{2}\right)=\left\{290^{\circ}, 30^{\circ}\right\}$ and some more isolated clicks. The second cluster may contain several closely spaced animals but on the whole at least two vocalising mammals can be numbered in this sequence. The mammal corresponding to the first cluster has a very clear pattern of decreasing elevation 
and azimuth in time. The second cluster is less obvious; there could be two animals close to each other. Further clustering and disentanglement of click series could be useful to obtain a better separation. From (c), elevation seems to indicate that there could be more than just two animals in the second cluster indeed elevation normally varies very little at large distances whereas well separated values of elevation $\left(>5^{\circ}\right)$ were found at a particular instant in time. Since the particular geometry makes it also less sensitive to small variation in azimuth, there could well be more than one animal in that cluster.

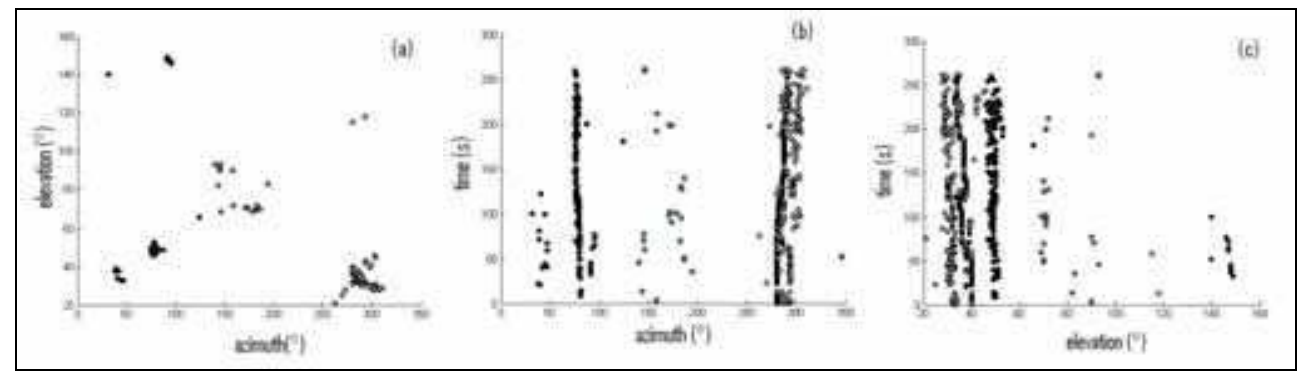

Fig. 6.6. Sperm whale tracking, 09th August 2005, 09pm

\section{Track 3: Dataset 18 ${ }^{\text {th }}$ August 2005, 11 pm}

760 sperm whale clicks were taken into account for localisation. One animal is clearly localised around $\left(\theta_{1}, \varphi_{1}\right)=\left\{110^{\circ}, 45^{\circ}\right\}$ and features a relatively stable elevation and a decreasing azimuth.

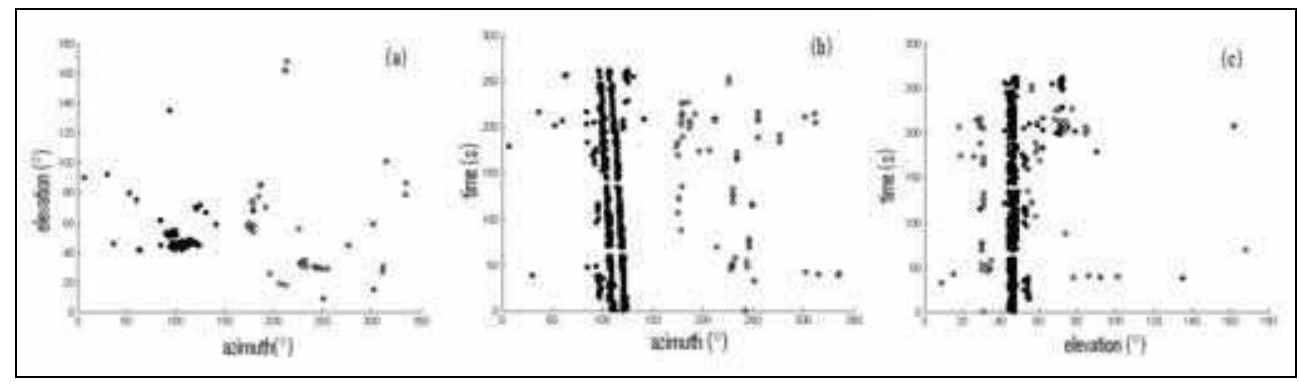

Fig. 6.7. Sperm whale tracking, $18^{\text {th }}$ August 2005, 11pm

\section{Track 4: Dataset 09th August 2005, 02 am}

701 impulsive sounds were taken into account for localisation. An experienced operator aurally identified them as being shipping impulsive sounds. Contrary to the tracking of sperm whales, the tracking of boats features a clear evolution of DOA during the available five minutes. This seems to confirm the fact that boats are localised since their speed is expected to be much faster than that of sperm whales. The first cluster around $\left(\theta_{1}, \varphi_{1}\right)=$ $\left\{100^{\circ}, 65^{\circ}\right\}$ corresponds to a source which starts to radiate around 150s. It features a slow but clear increase of both azimuth and elevation. The second cluster around $\left(\theta_{2}, \varphi_{2}\right)=\left\{275^{\circ}, 45^{\circ}\right\}$ corresponds to a source which radiates regularly during the 5 minutes of recording. It features a fast decrease of azimuth and a fast increase of elevation. 


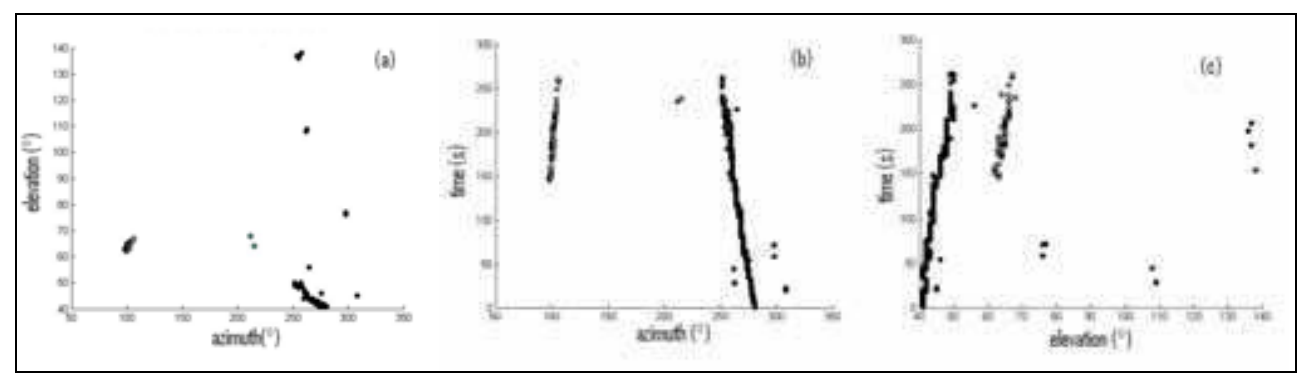

Fig. 6.8. Vessel tracking, 09th August 2005, 02am

\subsection{Discussion}

\section{Discussion on click-by-click localisation}

For space-time methods, two main reasons could explain the poor performance of the Capon and MuSiC algorithms which theoretically perform better than SRP. Firstly, both of these methods are extremely sensitive to the possible misestimation of the SCM (Krim \& Viberg, 1996). SCM is in particular difficult to estimate correctly for short duration signals. This problem appeared to be partly solved by hybrid methods. Secondly, these methods are sensitive to the amplitude mismatch caused by unknown differences in sensitivity of the hydrophones. This could be corrected for but only at the expense of additional computations, which are not developed here for conciseness. These corrections would also add to the respective processing times. Some of the hybrid methods presented in the next section seemed to demonstrate that this problem could be solved with reasonable processing times.

Among the space-time methods, the SRP algorithm, even though it is less sophisticated, seems to be the best compromise between accuracy and processing time (Houégnigan et al, 2010). Considering that calculations could be carried out much faster in parallel and on a dedicated computation platform, considering also commonly observed inter-click intervals for sperm whales between 0.5 and 2 seconds and the pauses been sequences of clicks (Wahlberg, 2002), an SRP implementation could be well-suited for real-time applications.

The interest of hybrid methods seems manifest from the results, which can be compared to those obtained with space-time methods while requiring less processing time. For example, SRP-ROTH was comparable to the non-hybrid EIG but took about a third of its time. In general the hybrid methods presented here (many other filters -and thus other hybridscould be considered) are also extremely profitable to the simple SRP algorithm. An interpretation of these results based on the nature of the filters used in the Generalised Cross-Correlation can be done.

(1) By filtering the signals, hybrids seem to construct a better estimation of the spatial correlation matrix. This estimate is not necessarily closer to the real spatial correlation matrix but more likely this estimate is more adapted to the nature of the spatial spectral estimators to which it is associated. Each of the estimators indeed uses a particular balance of noise and signal to achieve localisation, which is affected by the filters used for the Generalised Cross-Correlation.

The bad performance of the hybridised EIG, which relies on signal information by estimating the highest eigenvalue, is a sign that the signal components obtained after filtering are incorrect. The SCOT and ROTH filters, by taking into account coherence, 
blindly enhance spectral regions with high energy which may contain both noise and signal. The signal estimated by EIG-SCOT or EIG-ROTH is hence probably not only signal but a mixture of signal and noise, which leads to localisation errors. A filter more adapted to that algorithm could be imagined. On the contrary, since MuSiC and Capon rely on noise estimation, they location estimation is improved. Obviously, even though some noisy components are labelled as signal, the remaining noise components, those with low energy, are likely to contain less signal and hence to improve MuSiC and Capon.

(2) The filters have to be well adapted to the ambient noise present and to the levels of reverberation. It was for example noticed that the PHAT filter, frequently used for human speaker localisation was not well suited for data from the NEMO deep-sea observatory. More adaptive pre-filters could also be created. In general, in the use of hybrids one should be aware of the effects of each filter and of the modus operandi of each spectral estimator with regard to the spatial correlation matrix.

(3) The signals received on hydrophones resulting from broadband sound emitted by cetaceans are corrupted by noise and may feature an important dynamic range across the frequency bands, which makes estimation more difficult. Indeed, the contribution of weak signal components is likely to be underestimated whereas they could provide valuable information. Pre-whitening can reduce this dynamic range and is one of the capabilities of the SCOT and ROTH filters.

\subsection{Discussion on tracking}

Although they cannot be confirmed by sightings, the estimated tracks were consistent with what can be expected from a sperm whale. In five-minute sequences the bearing may not change drastically given the expected slow speed of sperm whales (such as 0.2 to $2.6 \mathrm{~m} / \mathrm{s}$ observed in Wahlberg, 2002) but coherent evolution of azimuth and elevation with time can be reconstructed. Track 6 shows that the localisation of vessels performs consistently without even proceeding to clustering. For sperm whales, additional clustering may add consistency to the results displayed but might as well discard valid isolated clicks. Already, the spatial separation abilities permitted to proceed to an estimation of the minimal number of vocalising mammals. The developed methods would benefit from being trained on data using a known moving source; this would permit to assess more precisely their performances.

\section{Conclusions}

\subsection{Ambient noise imaging to track non-vocalising sperm whales}

For a given and well-characterized signal, detection probabilities mostly depend on the background noise level. Before attempting the implementation of our passive approach in a specific area, it should be noted that ambient noise level statistics are the most limiting factor. We inferred from the literature that, in the band of interest, noise level was around 90 $\mathrm{dBrms}$ re $1 \mu \mathrm{Pa}$ for sea-state 1 and a $1-30 \mathrm{kHz}$ bandwidth. From our simulation results, energy-based detection thresholds would work until $1000 \mathrm{~m}$. Nonetheless, each increase of 6 $\mathrm{dB}$ in background noise level, which is far from unusual, would half the detection range, as most propagation spreading is spherical in our case, and would make the system unreliable due to the dependency on weather conditions. Advanced post-processing of the received low-level signals was not studied. The inherent spatio-temporal nature of sperm whale acoustics and behaviour requires the use of either stochastic or determinist signal processing 
to further increase the SNR. Statistical methods for ANI have been thoroughly studied in shallow water (Potter \& Chitre, 1996, 1999), but due to the numerous contextual differences, especially the limited number of active sources, it is likely that a stochastic approach would not be appropriate in our case. On the other hand, a determinist approach founded on proper modelling of source angular variability could prove robust. Among other welldocumented methods, passive 3D localization of active sperm whales could then provide triggering information to coherently sum up the silent whale's response and increase the SNR and compensate for the ambient noise variability.

The reported multi-pulse structure of (most probably) offaxis clicks was not simulated, due to our incapacity to infer a model of its three-dimensional properties. We hence limited our study to the propagation of the first main pulse. Yet, including this feature would not impact upon the received levels except in the rare cases of constructive or destructive overlaps. The greatest impact would more likely be on the 'fillup' of the time-space window with more high-energy pulses at the monitoring point, which may handicap the search for low level echoes in background noise. It is generally reported that the secondary pulses are rarely more than two or three and only appear at frequencies higher than 4 to $5 \mathrm{kHz}$ (see Figure 1). The whole signal duration may then increase to $20 \mathrm{msec}$ which results in a maximum $20 \times 8 \times 2=320 \mathrm{msec}$ time period. This is one-third of the search time window, for 8 vocal whales and taking direct, surface and bottom reflected signals to the buoy into account, at a rate of 1 click/whale/s.

In the usual case, detection rates would not be drastically altered. This paper would not be complete without a note on false alarm rates and how they would impact on a vessel's decision, as detectable echoes from the surface may often come from different sources, like a densely concentrated group of fish. At-sea experiments and real recordings may provide the relevant information to discriminate these other types of objects, e.g. by incorporating their monitored spatio-temporal and behavioural characteristics. Scattering was only modelled by surface and bottom reflection coefficients being altered depending on sea-state and bottom type, respectively. As a result, our scattering model only affects specular rays. Reverberation, e.g. nonspecular rays back-scattered from surface, bottom or deep scattering layers was not mentioned nor simulated. When propagating through a deep scattering layer, direct rays from source to target could also reach the receiver with interference scattered from the deep layer, attenuated by 40 to $50 \mathrm{~dB}$ (Jensen et al., 2000). Such attenuation could differ when deep scattering layers are at lower depth at night-time. During daytime, such layers tend to be at greater depths and would be further attenuated due to propagation loss. In either case, the resulting reverberations may interfere with the low-level echoes from silent whales. Similarly, modelling of surface and bottom scattering would provide important information on the interferences from the reverberated sources as a function of sea-state and time, since no detection will be possible if these are omnipresent, even for low scattering strengths. Even though we have shown that signals echoed from silent whales could be detectable at only low sea-states, when surface scattering may become negligible, bottom scattering strength could constantly interfere with and increase noise to critical levels. In this work, simulations accounted for a given number of vocalizing whales, each producing one direct, one surface reflected and one bottom-reflected ray to the receiver and to one silent whale, which in turn radiated the corresponding echoes modelled by one direct, one surface-reflected and one bottom-reflected ray to the receiver. In fact, these 12 resulting rays represent only one part of the real signal at the receiver, as all vocalizing whales would also scatter energy from other whales' clicks. In addition, 
simulations were limited to allow only one bottom and one surface reflection. Multiple reflections from vocalizing whales' clicks would originate weak signals of a similar order of magnitude as the simulated silent whale's echoes and should be discarded as well. So far, we have not studied how adding these additional scatterers and pathways could alter the current results, as the objective of this work was to study whether a signal excess from a silent whale near the surface could be measured. The raised ambiguity and false-alarm rates due to unpredicted and more complex pathways would probably call for a more advanced detector. As the primary task of the WACS is to localize active whales using an array of receivers, the resulting information could be used to perform forward modelling of the arrival structure, and then to compare this with observations to identify the anticipated replica arrivals. Echoed signals from silent whales could then be detected by a band-limited energy detector. In future work the authors hope to be able to simulate the same scenario with an unlimited number of reflections and enable back-scattering from active whales so that more complex detectors and matched field methods can properly be evaluated.

While this study is restricted to sperm whales, the ANI approach might progressively extend to wider possibilities, as large baleen whales passing through a wide pod of sperm whales are also to be detected, probably with higher contrast in the case of species such as fin and blue whales. Most large baleen whales only produce very low frequency sounds (most of the energy remains below $100 \mathrm{~Hz}$ ) that reverberate in a complex way in the SOFAR channel and mix with all types of low-frequency sources summing up to great sound pressure levels (Potter \& Delory, 1998). As a direct consequence, designing a permanent solution for passive localization of these whales is a difficult task and furthermore can be performed only with very wide aperture bottom-mounted arrays. The low and, at times, negative signal-to-noise ratios at relatively short range from the whales have motivated the specific development of advanced signal processing algorithms that have not yet been implemented and still need further development (Delory \& Potter, 1999; Delory et al., 1999). We believe that our approach could be an alternative worth considering in areas where sperm whale populations are geographically dense and stable over time. Furthermore, this method would have to be a complementary component of a more complex system like the previously described WACS in order to be viable and useful.

In conclusion, the results provided quantitative information as regards the implementation of a passive approach using sperm whale clicks as illuminating sources. Received levels are centred on ambient noise levels for low sea-states, motivating the use of beam-forming to raise signal levels and extract bearing information. Validation of the method introduced in this paper is essential before advanced signal enhancement techniques can be properly evaluated, leading to the prior necessity of performing experiments in the field. From a broader perspective, as permanent passive techniques based on natural acoustic energy would be probably less costly and less prejudicial to cetaceans than conventional active solutions the authors believe that they merit further investigation.

\subsection{Space-time and hybrid algorithms for the passive acoustic localisation of sperm whales and vessels}

This paper presented space-time estimators in a broadband frame and introduced novel hybrid methods. These developments could benefit the localisation of cetaceans emitting broadband sound, e.g. sperm whales, and can also be used for the localisation of vessels emitting broadband sound. When hybridised, basic space-time algorithms such as SRP were improved and performed as consistently as more sophisticated high-resolution estimators 
such as MuSiC or EIG. It was observed that hybrid high-resolution algorithms did improve the robustness of space-time high-resolution methods provided that pre-filtering is consistent with their treatment of noise and signal. All of these developments are always considered in the scope of a real-time processing frame in which at least SRP and hybrids would fit.

SRP-based localisation of series of detected impulsive sounds pre-classified as sperm whales or shipping noise permitted to construct consistent tracks of the radiating sources. The good spatial separation achieved by the algorithm permitted to obtain a first estimate of the number of sperm whales. For boats, the clarity of the tracks was higher while both the variance and the number of anomalies seemed drastically reduced probably due to the regular and mechanical aspect of sound radiation. In all cases, but especially for sperm whales, the developed methods would benefit from being tested on longer data sets, which may confirm the consistency of the track by displaying more complex motion patterns.

Future work will focus on the validation of the methods with known sound sources and on range estimation. Promising simulations showed that information on phase and amplitude contained in the spatial correlation matrix could permit an estimation of range. This approach may though require well-calibrated and equivalent sensors. Such an engineering requirement is highly constrained by technical costs; however it should be considered that such costs could be balanced by the gain of more precise results in localisation and enhanced capabilities for de-noising and sound enhancement. This may in turn be used to improve detection and classification. Another approach could consist in considering reflections for passive ranging. The association of the use of reflections and space-time or hybrid methods will be a challenging task and could bring powerful developments. Future work shall also include the influence of sound speed variation via an adequate insertion of sound speed profiles.

\section{References}

Alder-Frenchel, H.S. (1980). Acoustically derived estimate of the size distribution for a sample of sperm whales, Physeter catodon, in the western north Atlantic. Canadian Journal of Fisheries and Aquatic Sciences, 37, 2358-2361.

André, M. (1997.) El cachalote, Physeter macrocephalus, en las Islas Canarias. PhD Thesis. Universidad de Las Palmas de Gran Canaria.

André, M. \& Kamminga, C. (2000). Rhythmic dimension in the echolocation click trains of sperm whales: a possible function of identification and communication. Journal of the Marine Biological Association of the United Kingdom, 80, 163-169.

André, M., Delory, E. \& van der Schaar, M. (2004a). A passive acoustic solution to 3D whale monitoring. Brest, France: Sea-Tech Week.

André, M., Delory, E. \& van der Schaar, M. (2004b). A passive mitigation solution to the effects of human-generated sound on marine mammals. London: Policy on Sounds and Marine Mammals: an International Workshop.

André, M., Delory, E., van der Schaar, M. \& Castell, J.V. (2005). On the possibility of detecting and tracking echolocating whales by passive acoustics and ambient noise imaging. La Rochelle, France: Workshop on Active Sonar, 19th Conference of the European Cetacean Society. 
André, M., Johansson, A.T., Delory, E., van der Schaar, M. (2007). Foraging on squids: the sperm whale mid-range sonar. Journal of the Marine Biological Association of the United Kingdom. 2007, 87, 59-67.

André, M. (2009). The sperm whale sonar: monitoring and use in mitigation of anthropogenic noise effects in the marine environment. Nucl. Inst. Meth. Phys. Res. A 602, 262.

Buckingham, M.J., Potter, J.R. \& Epifanio, C.L. (1996). Seeing underwater with background noise. Scientific American, 274, 40-44.

Daziens, J.M. (2004). Assessing the performance of omni-directional receivers for passive acoustic detection of vocalizing odontocetes. Monterey, California: Naval Postgraduate School.

Delory, E. \& Potter, J.R. (1999). Transient, tonal, and background noise filtering with wavelet and cosine transforms. Journal of the Acoustical Society of America, 105, 1106.

Delory, E., Potter, J.R., Miller, C. \& Chiu, C.-S. (1999). Detection of blue whales A and B calls in the northeast Pacific Ocean using a multi-scale discriminant operator. Maui, Hawaii: .

Delory, E., André, M., Navarro Mesa, J.-L., Van der Schaar, M. (2007). On the possibility of detecting surfacing sperm whales at risk of collision using others' foraging clicks. J. Mar. Biol. Ass. U.K. (2007), 87, 47-58.

DiBiase, J. H., Silverman, H.F. and Brandstein, M.S. (2001). Microphone Arrays: Techniques and Applications, Springer-Verlag, 2001, pp. 157-180.

Dmochowski, P., Benesty, J. and Affes, S. (2007). Direction of arrival estimation using eigenanalysis of the parameterized spatial correlation matrix, in Proc. IEEE ICASSP, 2007, pp. I-1--I-4.

Goold, J. C. \& Jones, S. E. (1995). Time and frequency domain characteristics of sperm whale clicks. Journal of the Acoustical Society of America, vol. 98, pp. 1279-1291.

Griebel, S.M. \& Brandstein, M.S. (2001). Microphone array source localization using realizable delay vectors, in Proc. IEEE Workshop on Applications of Signal Processing to Audio and Acoustics, 2001.

Houégnigan L., Zaugg, S., van der Schaar, M., André, M. (2010). Space-time and hybrid algorithms for the passive acoustic localisation of sperm whales and vessels. Appl Acoust., doi:10.1016/j.apacoust.2010.05.017.

Jaquet, N., Dawson, S. \& Douglas, L. (2001). Vocal behavior of male sperm whales: why do they click? Journal of the Acoustical Society of America, 109, 2254-2259.

Johnson, D.H. (1982). The application of spectral estimation methods to bearing estimation problems, Proc. IEEE, vol. 70, 1982, pp. 1018-1028.

Krim, H. \& Viberg, M. (1996). Two decades of array signal processing research: The parametric approach", IEEE Signal Process. Mag. 13, 1996, pp. 67-94.

Makris, N.C. \& Cato, D.H. (1994). Using singing whales to track nonsingers. Journal of the Acoustical Society of America, 96, 3270.

Makris, N.C., Lai, Y.-S. \& Cato, D.H. (1999). Using broadband humpback whale vocalizations to locate nonvocal whales in shallow water. Journal of the Acoustical Society of America, 105, 993.

Møhl, B., Wahlberg, M., Madsen, P.T., Heerfordt, A. \& Lund, A., (2003). The monopulsed nature of sperm whale clicks. Journal of the Acoustical Society of America, 114, 11431154. 
Pavan, G., Hayward, T.J., Borsani, J.F., Priano, M., Manghi, M., Fossati, C. \& Gordon, J. (2000). Time patterns of sperm whale codas recorded in the Mediterranean Sea 1985-1996. Journal of the Acoustical Society of America, 107, 3487-3495.

Potter, J.R., Buckingham, M.J., Deane, G.B., Epifanio, C.L. \& Carbone, N.M. (1994). Acoustic daylight: preliminary results from an ambient noise imaging system. Journal of the Acoustical Society of America, 96, 3235.

Potter, J.R. \& Chitre, M. (1996). Statistical models for ambient noise imaging in temperate and tropical waters. Journal of the Acoustical Society of America, 100, 2738-2739.

Potter, J.R. \& Delory, E. (1998). Noise sources in the sea and the impact for those who live there. Singapore: Acoustic and Vibration, Asia' 98.

Potter, J.R. \& Chitre, M. (1999). Ambient noise imaging in warm shallow seas; second-order moment and model-based imaging algorithms. Journal of the Acoustical Society of America, 106, 3201-3210.

Riccobene, G. (2009). NEMO Collaboration. Long-term measurements of acoustic background noise in very deep sea, Nucl. Instr. and Meth. A. 604 S149-S157.

Richardson, W.J., Greene, C.R., Malme, C.L. \& Thomson, D.H. (1995). Marine mammals and noise. San Diego: Academic Press.

Schmidt, R. (1986). Multiple emitter location and signal parameter estimation, IEEE Transactions on Antennas and Propagation, Vol.34, No. 3, March 1986, pp:276 - 280.

Tucker, D.G. \& Glazey, B.K. (1966). Applied underwater acoustics. London: Pergamon Press Ltd.

Urick, R.J. (1996). Principles of Underwater Sound. Slough, UK: Peninsula Publishing.

Van der Schaar, M. \& André, M. (2006). An Alternative Sperm Whale (Physeter macrocephalus) Coda Naming Protocol. Aquatic Mammals 32(3), 370-373.

Wahlberg, M. (2002). The acoustic behaviour of diving sperm whales observed with a hydrophone array". Journal of Experimental Marine Biology and Ecology 281(1-2), pp. 53-62.

Wang, H. \& Kaveh, M. (1985). Coherent signal-subspace processing for the detection and estimation of angles-of-arrival of multiple wideband sources, IEEE Trans. Acoust. Speech Signal Process 33 4, August 1985, pp. 823-831.

Watkins, W. A. (1980). Sperm whale clicks, in Animal Sonar Systems, R.-G. B. a. J. F. Fish, Ed. New York: Plenum, 1980, pp. 283-290.

Serge Zaugg, S., van der Schaar, M., Houégnigan, L., Gervaise, C., André, M. (2010). Realtime acoustic classification of sperm whale clicks and shipping impulses. Appl Acoust, doi:10.1016/j.apacoust.2010.05.005.

Zimmer, W.M.X., Tyack, P.L., Johnson, M.P. \& Madsen, P.T., (2005). Three-dimensional beam pattern of regular sperm whale clicks confirms bent-horn hypothesis. Journal of the Acoustical Society of America, 117, 1473-1485.

Ziomek, L.J. (1995). Fundamentals of Acoustic Field Theory and Space-Time Signal Processing, CRC Press, 1995. 


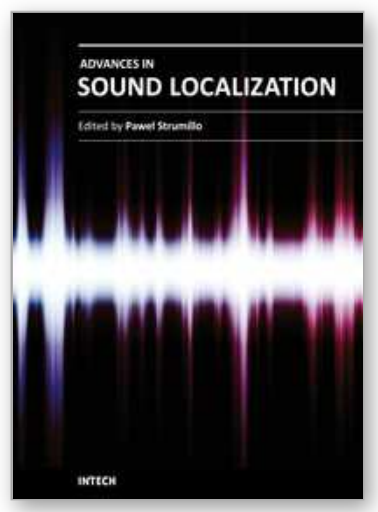

\author{
Advances in Sound Localization \\ Edited by Dr. Pawel Strumillo
}

ISBN 978-953-307-224-1

Hard cover, 590 pages

Publisher InTech

Published online 11, April, 2011

Published in print edition April, 2011

Sound source localization is an important research field that has attracted researchers' efforts from many technical and biomedical sciences. Sound source localization (SSL) is defined as the determination of the direction from a receiver, but also includes the distance from it. Because of the wave nature of sound propagation, phenomena such as refraction, diffraction, diffusion, reflection, reverberation and interference occur. The wide spectrum of sound frequencies that range from infrasounds through acoustic sounds to ultrasounds, also introduces difficulties, as different spectrum components have different penetration properties through the medium. Consequently, SSL is a complex computation problem and development of robust sound localization techniques calls for different approaches, including multisensor schemes, nullsteering beamforming and time-difference arrival techniques. The book offers a rich source of valuable material on advances on SSL techniques and their applications that should appeal to researches representing diverse engineering and scientific disciplines.

\title{
How to reference
}

In order to correctly reference this scholarly work, feel free to copy and paste the following:

Michel André, Ludwig Houégnigan, Mike van der Schaar, Eric Delory, Serge Zaugg, Antonio M. Sánchez and Alex Mas (2011). Localising Cetacean Sounds for the Real-Time Mitigation and Long-Term Acoustic Monitoring of Noise, Advances in Sound Localization, Dr. Pawel Strumillo (Ed.), ISBN: 978-953-307-224-1, InTech, Available from: http://www.intechopen.com/books/advances-in-sound-localization/localising-cetaceansounds-for-the-real-time-mitigation-and-long-term-acoustic-monitoring-of-noise

\section{INTECH}

open science | open minds

\section{InTech Europe}

University Campus STeP Ri

Slavka Krautzeka 83/A

51000 Rijeka, Croatia

Phone: +385 (51) 770447

Fax: +385 (51) 686166

www.intechopen.com

\section{InTech China}

Unit 405, Office Block, Hotel Equatorial Shanghai

No.65, Yan An Road (West), Shanghai, 200040, China 中国上海市延安西路65号上海国际贵都大饭店办公楼 405 单元

Phone: +86-21-62489820

Fax: +86-21-62489821 
(C) 2011 The Author(s). Licensee IntechOpen. This chapter is distributed under the terms of the Creative Commons Attribution-NonCommercialShareAlike-3.0 License, which permits use, distribution and reproduction for non-commercial purposes, provided the original is properly cited and derivative works building on this content are distributed under the same license. 